\title{
Observed Kinematic and Thermodynamic Structure in the Hurricane Boundary Layer during Intensity Change
}

\author{
KYLE AHERN \\ Florida State University, Tallahassee, Florida \\ MARK A. BOURASSA \\ Center for Ocean-Atmospheric Prediction Studies, Tallahassee, Florida \\ ROBERT E. HART \\ Florida State University, Tallahassee, Florida \\ JUN A. ZHANG \\ NOAA/AOML/Hurricane Research Division, and Cooperative Institute for Marine and Atmospheric Studies, \\ University of Miami, Miami, Florida \\ ROBERT F. ROGERS \\ NOAA/AOML/Hurricane Research Division, Miami, Florida
}

(Manuscript received 25 October 2018, in final form 3 May 2019)

\begin{abstract}
The axisymmetric structure of the inner-core hurricane boundary layer (BL) during intensification [IN; intensity tendency $\geq 20 \mathrm{kt}(24 \mathrm{~h})^{-1}$, where $1 \mathrm{kt} \approx 0.5144 \mathrm{~m} \mathrm{~s}^{-1}$ ], weakening [WE; intensity tendency $<-10 \mathrm{kt}$ $(24 \mathrm{~h})^{-1}$ ], and steady-state [SS; the remainder] periods are analyzed using composites of GPS dropwindsondes from reconnaissance missions between 1998 and 2015. A total of 3091 dropsondes were composited for analysis below 2.5-km elevation-1086 during IN, 1042 during WE, and 963 during SS. In nonintensifying hurricanes, the low-level tangential wind is greater outside the radius of maximum wind (RMW) than for intensifying hurricanes, implying higher inertial stability $\left(I^{2}\right)$ at those radii for nonintensifying hurricanes. Differences in tangential wind structure (and $I^{2}$ ) between the groups also imply differences in secondary circulation. The IN radial inflow layer is of nearly equal or greater thickness than nonintensifying groups, and all groups show an inflow maximum just outside the RMW. Nonintensifying hurricanes have stronger inflow outside the eyewall region, likely associated with frictionally forced ascent out of the BL and enhanced subsidence into the BL at radii outside the RMW. Equivalent potential temperatures $\left(\theta_{e}\right)$ and conditional stability are highest inside the RMW of nonintensifying storms, which is potentially related to TC intensity. At greater radii, inflow layer $\theta_{e}$ is lowest in WE hurricanes, suggesting greater subsidence or more convective downdrafts at those radii compared to IN and SS hurricanes. Comparisons of prior observational and theoretical studies are highlighted, especially those relating BL structure to large-scale vortex structure, convection, and intensity.
\end{abstract}

\section{Introduction}

The atmospheric boundary layer (BL) encapsulates a near-surface region where turbulent motions mix 3D kinematic and thermodynamic properties. For tropical cyclones (TCs), the fact that life and property inhabit the

\footnotetext{
Corresponding author: Kyle Ahern, kka09@my.fsu.edu
}

boundary layer (or TCBL, collectively) magnifies the BL's societal importance. In addition to strong sustained winds, powerful gusts, and persistent rainfall that accompany a TC, the loss of a TC's momentum through turbulent motions in the BL contributes to ocean wave generation and storm surge. Boundary layer processes (such as the frictional dissipation of momentum) are crucial to understanding a TC's evolution over time. 
Warm waters act as founts of energy for TC maintenance and growth-energy realized through latent and sensible heat fluxes at the air-sea interface (Riehl 1954). A well-developed TC resembles a thermodynamic engine whereby heat is extracted from the ocean surface, BL air is drawn toward the TC's center, and parcels ascend almost moist-neutrally from the top of the BL to the level of TC outflow aloft (Emanuel 1986). Ideally, the interactions between the ocean surface, TCBL, and TC "free atmosphere" should be well represented in forecasting tools. Aspirations to reduce TC forecast errors drive collective efforts to better our understanding of TCs and the TCBL (e.g., DeMaria et al. 2005; Rogers et al. 2006; Black et al. 2007; Chen et al. 2007; Rogers et al. 2013b).

From the premise that TC maintenance and intensity are at least partially dependent on heat fluxes, it follows that TCs are sensitive to exchange processes at the surface. The air-sea transfer of heat and moisture versus the frictional dissipation of momentum in TCs has elicited interest in the past-at times concerning the maximum (potential) intensity a TC could realize (e.g., Ooyama 1969; Rosenthal 1971; Emanuel 1988, 1995; Montgomery et al. 2010; Smith et al. 2014; Zhang and Emanuel 2016). Coefficients of enthalpy exchange $C_{k}$ and surface drag $C_{D}$ have been highlighted in some of the aforementioned research on potential intensity. Provided that $C_{D}$ is related to atmospheric momentum dissipation that acts to weaken a TC, and that $C_{k}$ is associated with supplying a TC's heat engine, it makes sense that the two coefficients are compared in efforts to understand and properly simulate steady-state TC intensity.

A TC's structure is also modulated by the BL. The interplay between a TC's primary and secondary circulations is imperative to changes in storm intensity (Ooyama 1969; Schubert and Hack 1982). Loss of absolute angular momentum $M_{a}$ in the TCBL results in an agradient force (Smith et al. 2009), where a reduction in total wind due to friction deflects flow toward the storm center and establishes a near-surface inflow layer. The inflow advects high- $M_{a}$ air toward the TC center, which induces a spinup tendency against frictional torque. Persing and Montgomery (2003) and Montgomery et al. (2006) highlighted instances where BL inflow may penetrate through the eyewall in examinations of hurricane "superintensity," whereby a hurricane achieves an intensity greater than a given theoretical limit for axisymmetrically balanced TCs [in reference to Emanuel $(1986,1995)]$. Parcels that enter a hurricane's eye can increase their equivalent potential temperature $\theta_{e}$ via air-sea fluxes and mixing with the eye's reservoir of high-entropy air before returning to the eyewall in low-level outflow. This process can add a source of internal energy to a TC's eyewall, affecting the secondary circulation and warm core structure (Shapiro and Willoughby 1982).

In studies by Zhang et al. (2011, hereafter Z11) and Barnes (2008), dropwindsonde observations exhibited marked dry and moist static stability near the top of the inflow layer, which may resist vertical displacements and protect the inflow from loss of entropy via entrainment. Barnes (2008) attributes the observed moist static stability to radial inflow undercutting rotational air near the high $-\theta_{e}$ core. In numerical simulations by Kepert et al. (2016), increased dry static stability near the top of inflow was attributed to diabatic processes associated with evaporating rainfall, and differential potential temperature $(\theta)$ advection due to near-surface vertical shear. Parcels ascending out of the insulated TCBL can carry relatively high $\theta_{e}$ air into the vortex aloft, which can affect conditional stability in the area of ascent.

Convergence in near-surface inflow favors frictionally forced ascent, which can translate through the TCBL top [or top of the inflow layer (Kepert 2013)]. Prior research suggests that BL convergence promotes regional favorability for deep ascent (Kepert 2013; Rogers et al. 2013a, 2015, 2016; Hazelton et al. 2017a; Zhang et al. 2017). Regarding TC intensification, much research has tackled the role of deep convection. Shapiro and Willoughby (1982) added heat sources as a proxy for convection to a simulated axisymmetric vortex, finding that intensification occurred if heat sources were sufficiently close to the radius of maximum winds (RMW). Pendergrass and Willoughby (2009) and Vigh and Schubert (2009) further emphasize the importance in position of heating, adding that storm strength and structure also bear roles in intensification [which was later confirmed observationally in Rogers et al. (2013a, hereafter R13a)]. Other studies examined vortex responses to asymmetric heating (Nolan and Montgomery 2002; Nolan and Grasso 2003; Nolan et al. 2007), concluding that intensification is approximately a symmetric response to azimuthally averaged heating; the asymmetric part of heating almost always served to weaken the mean vortex. However, modeling studies that added vorticity perturbations in lieu of heat sources (e.g., Montgomery and Kallenbach 1997; Möller and Montgomery 2000; Shapiro 2000) suggest that asymmetric convection can intensify a vortex via vortex Rossby waves, which can axisymmetrize potential vorticity generated through convection near the RMW.

In this paper, observations from global positioning system (GPS) dropwindsondes (dropsondes, sondes) are collected to examine the relationship between innercore TCBL structure and TC intensity change. Previous 
studies utilizing dropsondes have yielded a wealth of TC knowledge. LeeJoice (2000) used vertical profiles from dropsondes to analyze frictional inflow and near-surface thermodynamics, concluding that buoyancy may contribute considerably to eyewall inflow. Franklin et al. (2003, hereafter F03) examined soundings from several hurricanes to unveil mean vertical profiles of wind speed from the surface to flight level $(\sim 700 \mathrm{hPa})$, which exhibit a low-level maximum wind [at about $500 \mathrm{~m}$ above ground level (AGL)] in the eyewall. Below this wind "jet," a near-logarithmic reduction in wind speed with decreasing altitude is seen (Powell et al. 2003). Kepert (2006a, b) and Schwendike and Kepert (2008) used dropsonde data from four major hurricanes to analyze supergradient winds in and around the TCBL, concluding that the radial profile of gradient wind can diagnose supergradient flow. Bell and Montgomery (2008) studied Hurricane Isabel with dropsondes (among other data), finding strong near-surface inflow that allowed parcels to pierce the eyewall and enter a reservoir of high- $\theta_{e}$ air in the low-level eye. Zhang et al. (2013, hereafter Z13) and Z11 used composited soundings to investigate hurricane BL structure, including axisymmetric and asymmetric radial inflow layer structure and mixed-layer height. Z13 hypothesized that convective asymmetries seen in TCs (Black et al. 2002; Corbosiero and Molinari 2002, 2003; Chen et al. 2006; Reasor et al. 2013; DeHart et al. 2014; Hazelton et al. 2017a; Nguyen et al. 2017) are related to kinematic and thermodynamic asymmetries in the TCBL. The relation between BL kinematic and thermodynamic asymmetries (e.g., in near-surface $\theta_{e}$ and $\mathrm{BL}$ convergence) to convection was reverberated in a case study of the evolution of Hurricane Edouard in 2014 (Zawislak et al. 2016; Rogers et al. 2016). As these prior works have used dropsondes to reveal the lowertropospheric properties of TCs, many questions remain as to such properties in the context of intensity change. For example, it is unknown what structure the inflow layer tends to assume during TC intensification (or if a particular structure is assumed at all), and if that structure differs from the inflow layer of weakening TCs.

The crux of this endeavor is to contrast azimuthally averaged BL structures associated with hurricanestrength TCs that intensify versus those that weaken or maintain their intensity, thereby addressing some gaps in knowledge pertaining to TCBL structure during differing modes of intensity change. This work combines prior compositing methodologies for examining the TCBL (Z11; Z13) and TC intensity change (R13a) to produce a new, comprehensive dataset capable of comparing inner-core BL structure between groups of intensifying and nonintensifying (steady-state and weakening) TCs. Specifically, the BL structures of the primary and secondary circulation for intensifying and nonintensifying hurricanes are analyzed and compared. Thermodynamic BL properties are similarly examined (e.g., for comparisons of BL moist entropy). Alongside the existing literature, the results of this analysis should add some clarity regarding the nature of an evolving hurricane's BL. The findings herein may be used to verify or improve our representation of the TCBL in numerical frameworks, or as additional guidance for intensity forecasting.

\section{Methodology}

\section{a. Data}

GPS dropwindsonde data act as the principal component of this composite analysis. Developed by the National Center for Atmospheric Research (NCAR), these instruments have been deployed regularly in TC reconnaissance missions since 1998 (Hock and Franklin 1999). Dropsondes have a near-surface fall speed of $11-$ $12 \mathrm{~m} \mathrm{~s}^{-1}$ and a sampling rate of $2 \mathrm{~Hz}$, yielding a vertical resolution of about $5 \mathrm{~m}$ (F03). Each sample provides readings of location, pressure, air temperature, relative humidity, wind direction and speed, vertical velocity, and geopotential altitude. Estimated typical errors associated with NCAR dropsondes are $1 \mathrm{hPa}$ for pressure, $0.2^{\circ} \mathrm{C}$ for temperature, $<5 \%$ for relative humidity, and $0.5-2.0 \mathrm{~m} \mathrm{~s}^{-1}$ for wind. Hock and Franklin (1999) describe the background and utility of the NCAR GPS dropsonde.

For this work, soundings are collected from the Hurricane Research Division's (HRD) storm pages archives $^{1}$ to construct composites of TCBL inner-core structure in Atlantic hurricanes from 1998 to 2015. Changes in hurricane intensity separate data into intensifying (IN), weakening (WE), and steady-state (SS) composites. All dropsonde data utilized in this work undergo initial processing and quality control using the NCAR's Atmospheric Sounding Processing Environment (ASPEN) program. ${ }^{2}$ In total, 12045 soundings were processed, although not all soundings are utilized here due to data filtering in the compositing technique. To our knowledge, we have considered all available Atlantic sonde data from flights recording stepped frequency microwave radiometer data (SFMR; Uhlhorn and Black 2003; Uhlhorn et al. 2007; Klotz

\footnotetext{
${ }^{1}$ Accessed via http://www.aoml.noaa.gov/hrd/data_sub/hurr.html.

${ }^{2}$ Accessed via https://www.eol.ucar.edu/software/aspen.
} 
and Uhlhorn 2014) between 1998 and 2015, and these composites are the largest of their kind. The SFMR data are necessary to establish the radial coordinate of this analysis, which is radial distance normalized by the radius of maximum winds detected at the surface (i.e., $r_{\star} \equiv r / \mathrm{RMW}$ ). Following similar research that blends data from multiple storms (e.g., Z11; Rogers et al. 2012, 2013b; Z13), this normalization is applied to account for differences in storm size. However, the RMW data derived here likely differ from flight-level RMW data used in other studies, which we would expect to be generally greater than surface wind-based RMW in relation to eyewall slope (Rogers and Uhlhorn 2008; Stern et al. 2014; Hazelton et al. 2015).

\section{b. Compositing technique}

All data are reviewed to ensure that they are suitable for this analysis. GPS dropsonde data from sampled storms are eligible for compositing if, at the time of observation:

- The storm center and sonde are located over water,

- the storm is tropical and of hurricane intensity, and

- all required data for composite sorting and positioning are available; these include data for intensity tendency, track, and RMW.

A storm's center at a given time is defined via interpolation between 2-min storm track data from connected flight-level wind center fixes (Willoughby and Chelmow 1982). If a dropsonde or its associated storm's center are located over land [using a landmask from ERA-Interim data (Dee et al. 2011)] at any time during descent, the dropsonde is omitted from analysis. To determine if a sampled system is both tropical and of sufficient intensity, we refer to the revised Atlantic hurricane database (HURDAT2; Landsea and Franklin 2013), which provides "status of system" information (tropical, extratropical, etc.) and maximum sustained winds data ( $V_{\max }$, interchangeable with "intensity"). If either of the two HURDAT2 data records straddling a dropsonde sample in time suggest that the sampled storm is not tropical, the sample is ineligible for compositing. A dropsonde datum is removed from compositing if neither of the temporally neighboring HURDAT2 records indicate $V_{\max }>64 \mathrm{kt}\left(1 \mathrm{kt} \approx 0.5144 \mathrm{~m} \mathrm{~s}^{-1}\right)$. We limit our composites to include only samples from hurricanes, thereby reducing representation of tropical systems that have little or no organized vortex structure.

Eligible dropsonde data are sorted and gridded to cylindrical spaces based on the following:

- Intensity change (IN, SS, or WE),

- radial position relative to the TC center, normalized by RMW $\left(r_{\star}=r / \mathrm{RMW}\right)$,
- azimuth relative to the heading of environmental wind shear imposed on the TC, and

- height above the surface $(z)$.

Although data are averaged azimuthally for the purposes of analysis in section 4, we use the $850-200-\mathrm{hPa}$ shear parameter from SHIPS output (DeMaria et al. 2005) to calculate shear-relative azimuth (consistent with R13a; Z13; Reasor et al. 2013; DeHart et al. 2014) for a climatology of the composited data in section 3 .

HURDAT2 intensity data are consulted to determine the mode of intensity change of a given storm in time. The composite identifier associated with an observation is found by taking the most recent HURDAT2 records backward and forward in time, and then calculating the intensity tendency over the time between those two records. Following R13a, we define intensity change categories as follows:

- Intensifying (IN): $\frac{\Delta V_{\max }}{\Delta t} \geq \frac{20 \mathrm{kt}}{24 \mathrm{~h}}$

- Steady-state (SS): $\frac{20 \mathrm{kt}}{24 \mathrm{~h}}>\frac{\Delta V_{\max }}{\Delta t} \geq-\frac{10 \mathrm{kt}}{24 \mathrm{~h}}$,

- Weakening (WE): $\frac{\Delta V_{\max }}{\Delta t}<-\frac{10 \mathrm{kt}}{24 \mathrm{~h}}$.

Radial positions of dropsonde data, $r$, are found by calculating great-circle distance between data positions in the vertical (to account for instrument translation during descent) and 2-min storm track data (Willoughby and Chelmow 1982). Radius is divided by the nearest calculated RMW on record to yield normalized radius $r_{\star}$. Surface wind data from SFMR (Uhlhorn and Black 2003; Uhlhorn et al. 2007; Klotz and Uhlhorn 2014) onboard research and reconnaissance aircraft are used to compile a RMW database. The SFMR measures nadir brightness temperatures in six C-band frequencies at a nominal rate of $1 \mathrm{~Hz}$, using a geophysical model function relating excess surface emissivity and wind speed to produce surface wind speed estimates along a flight path. Here, we use a 60-s box smoother on SFMR surface wind data to reduce noise and the potential for transient gustiness to be interpreted as the maximum sustained wind speed.

During a flight, the aircraft can make several passes through a hurricane's eyewall. From each such pass, a SFMR-based determination of RMW is feasible provided the data exist. To find the RMW for a given pass, first we make a liberal guess at the annular or ringlike region containing the RMW (schematic shown in Fig. 1). The region's inner wall $\left(R_{\text {in }}\right)$ is defined using either a minimum distance or wind speed threshold (hence the region becomes "ringlike," ideal for hurricanes with calm eyes), and the region's outer border $\left(R_{\text {out }}\right)$ is defined using some maximum distance threshold. The first-guess region assumes an inner wall defined by the $15 \mathrm{~m} \mathrm{~s}^{-1}$ wind speed contour within $R_{\text {out }}$, which is $70 \mathrm{~km}$ by default. When the aircraft enters this region through $R_{\text {out }}$, we begin searching 

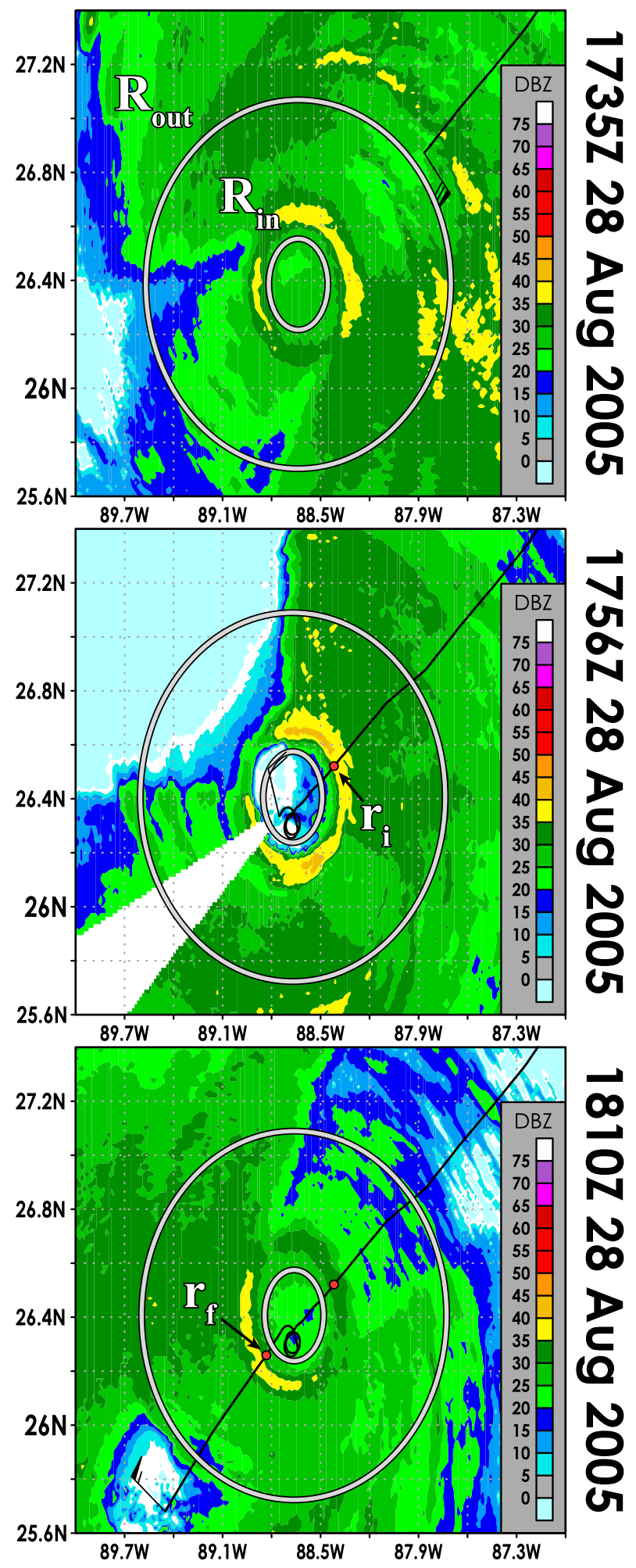

FIG. 1. Schematic depiction of extracting RMW data using Hurricane Katrina for example [lower fuselage radar reflectivity (dBZ) shaded]. The black line is the aircraft flight path, and the barb represents flight-level wind speed (kt) and direction. Ovals ( $R_{\text {out }}$ and $\left.R_{\text {in }}\right)$ are borders of the area where RMW searches for the maximum surface wind speed and its great-circle distance from the TC center $\left(r_{i}\right)$. As the aircraft exits the searching region through $R_{\mathrm{in}}, r_{i}$ is recorded. When the aircraft reenters the region via $R_{\text {in }}$, we scan again for the radius and time where surface wind is maximized $\left(r_{f}\right.$ and $t$, respectively). Once the aircraft leaves the region through $R_{\text {out }}$, the RMW at time $t$ is determined:

$$
\mathrm{RMW}=\frac{r_{i}+r_{f}}{2} .
$$

As an example, Fig. 2 shows the time series of SFMR surface wind data and radius associated with the flight in Fig. 1, along with times when $r_{i}$ and $r_{f}$ are recorded. All 451 initial (and subsequent) guesses at the RMW based on surface wind data are checked by hand for veracity, and the RMW is recalculated with a modified search region if necessary (e.g., if a valid maximum surface wind evidently lies outside $R_{\text {out }}$, or if the inner wind speed threshold fails). If a dropsonde's launch time is more than $12 \mathrm{~h}$ separated from the nearest RMW on record, it is discarded as the RMW could change substantially in that time period (cutoffs of 24,18 , and $6 \mathrm{~h}$ were also used; the results were robust to these adjustments).

Finally, sounding data with known $r \star$ and $z$ are sorted into appropriate composite spaces (IN, WE, or SS). We limit our analysis to observations at or below $2.5-\mathrm{km}$ elevation and at $r_{\star} \leq 3.0$. These composite spaces have a radial resolution of $0.25 \mathrm{RMW}$, and a vertical resolution of $25 \mathrm{~m}$. First, we will detail the resulting structures of data to aid composite interpretation. Our analysis of mean axisymmetric structures in each composite will begin with a kinematic focus, highlighting properties of the tangential and radial flow. We convert Earthrelative wind measurements to storm-relative tangential and radial winds by subtracting storm motion from the Earth-relative vectors and transposing the resulting vector field to an azimuthal equidistant projection (Snyder 1987) centered on the hurricane [detailed in Ahern and Cowan (2018)]. Examinations of thermodynamic structure will follow the kinematic analysis.

\section{Data climatology}

The launch position of each composited dropsonde is plotted in Fig. 3. Sampling occurs primarily in the western Atlantic basin, the Gulf of Mexico, and the Caribbean Sea. In the Caribbean Sea and southwestern

occur. (top) Scanning for $r_{i}$ begins after the aircraft enters the area via $R_{\text {out, }}$ (middle) $r_{i}$ is recorded after plane exits the area through $R_{\mathrm{in}}$; searching for $r_{f}$ will start when the plane reenters the area. (bottom) Searching for $r_{f}$ ends, and RMW is calculated. 


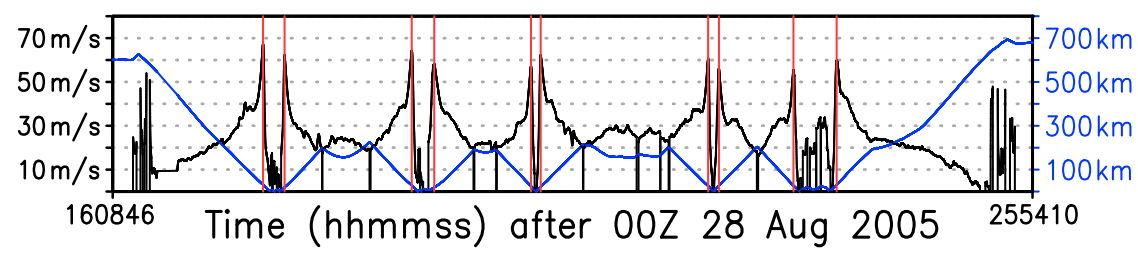

FIG. 2. Time series of 60-s averaged surface wind speed (black, left axis) and great circle distance from interpolated 2-min track position (blue, right axis), taken from the stepped frequency microwave radiometer (SFMR) on board NOAA aircraft 43RF during its mission into Hurricane Katrina on 28 Aug 2005 (same flight in Fig. 1). Times of recorded $r_{i}$ and $r_{f}$ values are marked with red lines.

Gulf of Mexico, most dropsonde launches are associated with IN. This is compared to the relatively diverse distribution of intensity change in the Gulf of Mexico north of $25^{\circ} \mathrm{N}$ and off the eastern U.S. seaboard [where influences of land, large-scale interaction, and lower sea surface temperatures (SSTs) are more prevalent].

Composited dropsonde launch positions relative to the environmental vertical shear (DeMaria et al. 2005) and RMW are shown in Fig. 4. In general, launches are clustered about the storm center, with high spatial density of launches for $r_{\star} \leq 1.5$ compared to outside this region. Soundings are somewhat more concentrated left of the vertical wind shear vector, particularly for $r_{\star} \leq 1.5$. All shear-relative quadrants are well sampled, so resulting azimuthally averaged structures will not be heavily skewed toward structures specific to one quadrant. These sampling traits persist regardless of subsampling based on intensity change.

A total of 3091 from an original pool of 12045 qualitycontrolled Atlantic dropsondes $(25.7 \%)$ comprise our composite datasets, with 1086 (963/1042) dropsondes launched when a storm was intensifying (steady-state/weakening). A summary highlighting the storms in which these dropsondes are launched and their distribution into composites, including best-track intensity and RMW ranges during dropsonde sampling, is provided in Table 1. Of the 50 hurricanes sampled, the cases contributing the most dropsondes overall are Hurricanes Rita in 2005 (212 sondes), Ike in 2008 (191), Irene in 2011 (190), Earl in 2010 (184), Isabel in 2003 (176), and Sandy in 2012 (155). Hurricanes Earl and Ike contribute the most launches to the IN composite (78 and 74 sondes, respectively). In the WE composite, Hurricanes Rita (138) and Isabel (96) are associated with the most dropsonde launches. Irene (106) and Bonnie in 1998 (138) contribute the most sonde launches to the SS composite.

Table 2 lists several environmental and storm characteristics, averaged across composited dropsondes at launch time. In the mean, IN storms are associated with the smallest RMWs, weakest deep-layer shear, and highest SSTs and environmental low-level relative humidity (RH). WE hurricanes, by comparison, have the strongest deep-layer shear, coldest SSTs, and least low-level RH. Past TC data climatologies in Kaplan and DeMaria (2003) and Hendricks et al. (2010) have found similar characteristics, although the magnitudes of fields like deep-layer shear are sometimes notably different. For example, the deep-layer shear calculated in Kaplan and DeMaria (2003) for nonrapidly intensifying TCs is comparable to our SS composite, but Hendricks et al. (2010) found stronger deep-layer shear for all Atlantic TCs regardless of stratification by intensity change. These differences highlight some sensitivity of the environmental climatology to the definitions used for modes of intensity change, as well as the data utilized to represent the environment itself.

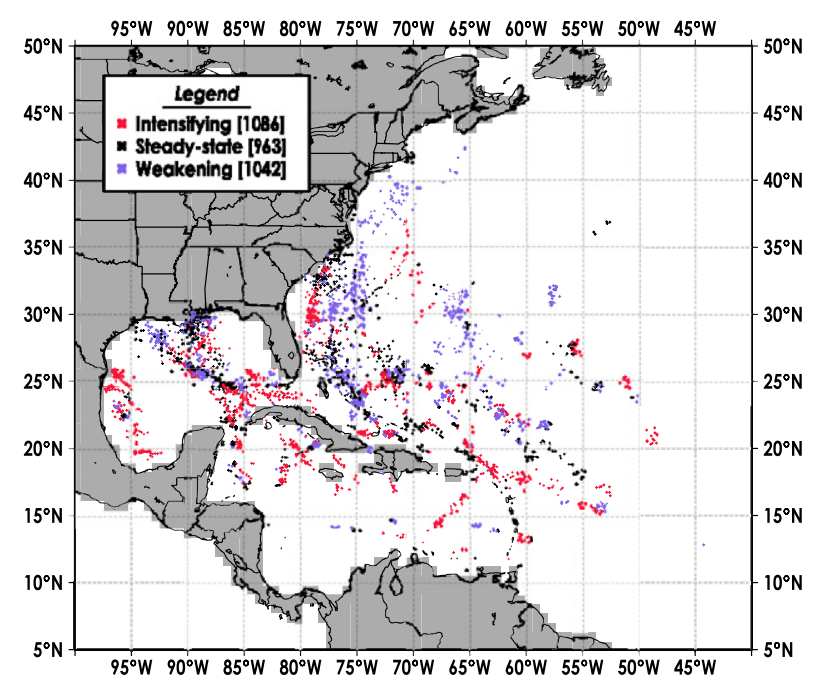

FIG. 3. Launch positions of all composited dropsondes for Atlantic hurricanes during the 1998-2015 period. The color of each dot represents the evolutionary identifier associated with a dropsonde at launch time (summarized in the figure legend). The amount of dropsondes utilized in each composite is contained in brackets next to each descriptor in the legend. Landmask for determining whether dropsonde data or storm centers were over land (and thus discarded) is colored in gray. 

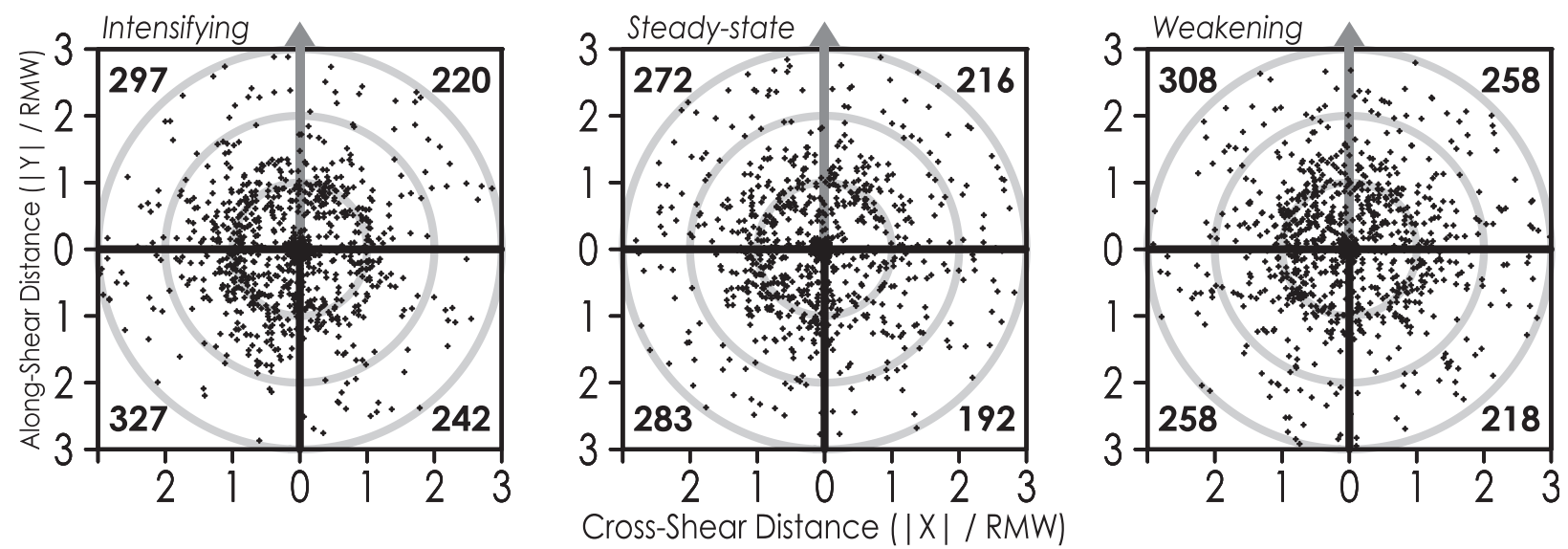

FIG. 4. Composited dropsonde launch positions on the horizontal plane for (left) intensifying, (middle) steady-state, and (right) weakening Atlantic storms. The $x$ and $y$ distances from the storm center are expressed in multiples of detected RMW. Concentric dividers represent contours of $r_{\star}$ with an interval of 1 . The bold gray vector represents the environmental wind shear as determined from SHIPS. The number in the corner of each shear-relative quadrant represents the number of sondes launched in that quadrant.

With a vast array of cases contributing data to the composites, the heterogeneity of information across composite space should also be considered. A composite representation of the TCBL (or TCs in general) loses meaning if the included storms are unique and contribute data unevenly in space. We will examine this problem by decomposing composited system and environmental data in space. Figure 5 breaks down the frequency of storm intensity during dropsonde launches as a function of $r_{\star}$, expressed as a percentage of total launches within the given radial bin. In the IN composite, samples at $r_{\star}>2$ are generally associated with weaker storms $(60-70 \mathrm{kt})$ compared to inner radii-hurricanes of this intensity account for more than $40 \%$ of data in some bins at these outer radii. Dropsondes from the SS composite originate mostly from hurricanes with $V_{\max }<120 \mathrm{kt}$, with sampling maxima near $100 \mathrm{kt}$ in most radial bins. Sondes from the WE group are taken from a more diverse range of intensities; sampling maxima are seen in the 135140 -kt intensity bin for $r \star$ between 0.75 and 1.5 , and in the 60-70-kt intensity range for $r \star$ between 1.75 and 2.75. The IN and WE composites both exhibit a tendency for relatively low intensity storms to be sampled at greater radii. Assuming the radial profile of wind associated with each sampled case has similar "peakedness," this suggests that the resulting tangential winds of IN and WE composites may be biased toward looking more peaked.

The spatial sampling trends of at-launch deeplayer wind shear (as determined from SHIPS) are shown in Fig. 6, framed similar to Fig. 5. For IN sondes, relative sampling frequency is mostly amplified between 6 and $21 \mathrm{kt}$, except at $r_{\star}>2.5$ where sampling maxima occur in the 30-33-kt range. The SS composite has a larger range of shear magnitudes across $r_{\star}$, with most sondes launched when the shear is between 6 and $30 \mathrm{kt}$. A small amount of steadystate dropsondes at outer radii are launched when the shear is quite strong ( $\geq 42 \mathrm{kt})$. The WE composite has a similar signal of strong shear at outer radii, and most WE sondes are launched when the shear magnitude is 9-27 kt. With respect to shear, there appears to be less sampling heterogeneity across $r \star$, so sampling biases across radius are not expected to affect composite results substantially. However, sampling biases may influence comparisons between composites due to the tendency for shear to be stronger in nonintensifying cases.

\section{Axisymmetric analysis}

To calculate a given field, we sum all measurements of the variable in each $\left(r_{\star}, z\right)$ bin, and then divide through by the number of observations summed from each bin (i.e., the mean in each bin). The frictional inflow layer, which is referenced throughout this analysis, is defined using a normalized and smoothed radial velocity field $u_{\mathrm{sm}, \star}=u_{\mathrm{sm}} /\left|u_{\min }\right|$, where $u_{\mathrm{sm}}$ is storm-relative radial velocity smoothed 5 times using a 1-2-1 filter and $\left|u_{\min }\right|$ is the peak composited inflow magnitude. The inflow layer is defined below $z=1.5 \mathrm{~km}$ where $u_{\star} \leq-0.1$, which is similar to the definition used in Z11.

\section{a. Primary circulation}

Normalized radius-height cross sections of stormrelative tangential wind $v$ are shown for all composites 
TABLE 1. List of Atlantic hurricanes included in composites. Number of dropsondes used is shown, as well as the amount of dropsondes launched under each intensification identifier. Ranges of best track storm intensity and SFMR-detected radius of maximum winds at times of observation are disclosed.

\begin{tabular}{|c|c|c|c|c|c|c|c|}
\hline Name & Year & All sondes & IN sondes & SS sondes & WE sondes & Sampled $V_{\max }$ & Sampled RMW \\
\hline Rita & 2005 & 212 & 62 & 12 & 138 & $60-146$ & $14.6-39.7$ \\
\hline Ike & 2008 & 191 & 74 & 98 & 19 & $65-115$ & $9.3-113.2$ \\
\hline Irene & 2011 & 190 & 22 & 106 & 62 & $65-105$ & $18.3-112.6$ \\
\hline Earl & 2010 & 184 & 78 & 30 & 76 & $60-125$ & $16.5-67.6$ \\
\hline Isabel & 2003 & 176 & 50 & 30 & 96 & $130-140$ & $25.8-41.8$ \\
\hline Sandy & 2012 & 155 & 33 & 61 & 61 & $62-95$ & $26-89.6$ \\
\hline Bonnie & 1998 & 146 & 0 & 138 & 8 & $95-100$ & $56.3-67.7$ \\
\hline Gonzalo & 2014 & 115 & 47 & 23 & 45 & $64-125$ & $6.6-49.5$ \\
\hline Ophelia & 2005 & 108 & 47 & 0 & 61 & $55-71$ & $14.8-100.1$ \\
\hline Frances & 2004 & 98 & 21 & 41 & 36 & $85-125$ & $18.5-66.5$ \\
\hline Gustav & 2008 & 95 & 34 & 38 & 23 & 59-126 & $14.7-49.8$ \\
\hline Arthur & 2014 & 90 & 50 & 12 & 28 & $60-85$ & $24.2-79.8$ \\
\hline Isaac & 2012 & 85 & 42 & 42 & 1 & $69-70$ & $36.5-88.3$ \\
\hline Ivan & 2004 & 79 & 1 & 51 & 27 & $105-140$ & $14-49.9$ \\
\hline Katrina & 2005 & 79 & 7 & 18 & 54 & $100-150$ & $26.6-64.4$ \\
\hline Bill & 2009 & 66 & 18 & 29 & 19 & $73-115$ & $27.2-44.1$ \\
\hline Joaquin & 2015 & 64 & 27 & 6 & 31 & $68-135$ & $20.9-47.1$ \\
\hline Lili & 2002 & 63 & 52 & 11 & 0 & $65-125$ & 14-31.6 \\
\hline Ingrid & 2013 & 61 & 34 & 7 & 20 & $60-72$ & $14-36.7$ \\
\hline Edouard & 2014 & 61 & 26 & 12 & 23 & $72-102$ & $18.4-42.5$ \\
\hline Georges & 1998 & 58 & 25 & 17 & 16 & $78-135$ & $19.1-68.6$ \\
\hline Wilma & 2005 & 53 & 37 & 8 & 8 & $85-110$ & $51.4-61.6$ \\
\hline Dennis & 2005 & 52 & 44 & 0 & 8 & $75-130$ & $14.1-26.2$ \\
\hline Helene & 2006 & 46 & 13 & 16 & 17 & 80-101 & 21.6-94.4 \\
\hline Danielle & 1998 & 45 & 0 & 24 & 21 & $65-70$ & $32.9-43.1$ \\
\hline Ida & 2009 & 42 & 31 & 3 & 8 & $60-90$ & $22.3-34$ \\
\hline Tomas & 2010 & 42 & 20 & 2 & 20 & $60-85$ & $21-53.7$ \\
\hline Isidore & 2002 & 40 & 0 & 40 & 0 & $\sim 110$ & $18.2-24.5$ \\
\hline Paloma & 2008 & 37 & 20 & 10 & 7 & $65-125$ & $10.6-29.1$ \\
\hline Omar & 2008 & 35 & 33 & 0 & 2 & 60-109 & $13.5-27.6$ \\
\hline Paula & 2010 & 34 & 5 & 16 & 13 & $65-90$ & $10.3-20.1$ \\
\hline Dolly & 2008 & 32 & 32 & 0 & 0 & $60-71$ & $25.2-40.2$ \\
\hline Fabian & 2003 & 32 & 6 & 14 & 12 & $105-120$ & $19.5-31.6$ \\
\hline Alex & 2010 & 29 & 23 & 6 & 0 & $65-81$ & $14.3-24.4$ \\
\hline Jeanne & 2004 & 28 & 0 & 28 & 0 & 85-105 & $28.7-59.9$ \\
\hline Karl & 2010 & 24 & 24 & 0 & 0 & $56-86$ & $17.3-28.4$ \\
\hline Igor & 2010 & 21 & 0 & 0 & 21 & $69-90$ & $57.2-89.6$ \\
\hline Felix & 2007 & 21 & 11 & 3 & 7 & $62-150$ & $9.9-21.3$ \\
\hline Rina & 2011 & 16 & 0 & 8 & 8 & 79-100 & $17-22.8$ \\
\hline Rafael & 2012 & 15 & 7 & 2 & 6 & $60-80$ & $24.7-74.1$ \\
\hline Danny & 2015 & 15 & 0 & 0 & 15 & $64-65$ & $11.4-15.2$ \\
\hline Katia & 2011 & 14 & 0 & 0 & 14 & 93-104 & $80.6-82.5$ \\
\hline Danielle & 2010 & 14 & 8 & 0 & 6 & $98-113$ & $17.8-62.9$ \\
\hline Kyle & 2008 & 12 & 11 & 1 & 0 & $60-70$ & $25.4-43.4$ \\
\hline Ernesto & 2012 & 5 & 5 & 0 & 0 & $61-68$ & $26.3-37.2$ \\
\hline Leslie & 2012 & 3 & 0 & 0 & 3 & $60-61$ & $\sim 95.1$ \\
\hline Fay & 2014 & 3 & 3 & 0 & 0 & $60-61$ & $\sim 95.2$ \\
\hline Kate & 2015 & 3 & 3 & 0 & 0 & $62-63$ & $\sim 18.3$ \\
\hline Karen & 2007 & 1 & 0 & 0 & 1 & $61-62$ & $\sim 29.6$ \\
\hline Bertha & 2008 & 1 & 0 & 0 & 1 & $66-67$ & $\sim 70.8$ \\
\hline
\end{tabular}

in Fig. 7. Regardless of stratification by intensity change, a low-level wind jet appears within $r_{\star}=(0.75$, 1.5], which is situated above $z \approx 250 \mathrm{~m}$ and extends up to $z \approx 1250 \mathrm{~m}$. This wind jet is similar to the mean wind speed profiles in hurricane eyewalls portrayed by F03, which found the strongest eyewall winds to be near $500 \mathrm{~m}$ above the surface. Aside from the effects of sampling biases, F03 suggest these low-level wind maxima are a consequence of warm-core cyclone structure. The jet magnitude is weakest in the IN group $\left(\sim 48 \mathrm{~m} \mathrm{~s}^{-1}\right.$ 
TABLE 2. Mean environmental conditions at launch of dropsondes in IN, SS, and WE composites. Standard deviations are given in parentheses. Shear, SST, and low-level relative humidity data are pulled from SHIPS data.

\begin{tabular}{lcll}
\hline \hline \multicolumn{1}{c}{ Quantity } & \multicolumn{1}{c}{ IN } & \multicolumn{1}{c}{ SS } & \multicolumn{1}{c}{ WE } \\
\hline $\begin{array}{l}850-200-h P a \text { shear } \\
\left(\mathrm{m} \mathrm{s}^{-1}\right)\end{array}$ & $7.66(4.31)$ & $8.97(5.13)$ & $9.76(5.12)$ \\
SST $\left({ }^{\circ} \mathrm{C}\right)$ & & & \\
$850-700-h P a$ relative & $68.95(7.64)$ & $67.61(5.68)$ & $66.70(6.36)$ \\
$\quad$ humidity $(\%)$ & & & \\
RMW $(\mathrm{km})$ & $34.57(19.17)$ & $43.75(22.60)$ & $41.15(21.23)$ \\
Intensity $V_{\max }\left(\mathrm{m} \mathrm{s}^{-1}\right)$ & $44.41(12.31)$ & $49.96(10.08)$ & $51.92(12.76)$ \\
Storm latitude $\left({ }^{\circ} \mathrm{N}\right)$ & $23.21(4.60)$ & $25.51(4.34)$ & $26.89(5.13)$ \\
Storm longitude $\left({ }^{\circ} \mathrm{W}\right)$ & $76.61(11.99)$ & $77.07(10.20)$ & $75.44(11.04)$ \\
\hline
\end{tabular}

at $500-700 \mathrm{~m})$ and strongest in the WE group $\left(\sim 53 \mathrm{~m} \mathrm{~s}^{-1}\right.$ at $375-900 \mathrm{~m})$. The jet seen in the SS group has a magnitude between the other groups, with $v \approx 51 \mathrm{~m} \mathrm{~s}^{-1}$ between 400 and $825 \mathrm{~m}$ AGL. Tangential wind maxima are concentrated near the RMW, with a general sloping slightly outward with height. Below $v$ maxima, $v$ decreases rapidly with decreasing height, in agreement with prior studies (e.g., F03; Powell et al. 2003; Z11). At greater radii from the RMW $\left(r_{\star}>1.75\right)$, the IN group's tangential wind field is weaker compared to nonintensifying groups.

Composite differences in $v$ are partly due to sampling biases in $V_{\max }$, which is plotted in Fig. 8. Regarding perceived structural differences in $v$ between the composite groups, sampled storm intensities should be taken into account. For example, the tangential wind jet seen in the WE composite group is apparently stronger than the jet from the IN composite group, but this difference is a function of the sampled storms' intensities; on average, eyewall observations in WE are associated with storms of greater intensity $\left(54-57 \mathrm{~m} \mathrm{~s}^{-1}\right)$ compared to eyewall measurements in IN $\left(48-54 \mathrm{~m} \mathrm{~s}^{-1}\right)$. This problem also applies within each composite, as the average intensity during eyewall sampling is generally greatest compared to sampling elsewhere.

The radial variation of $v$ (or a suitable analog) can be used to interpret the inertial stability of the vortex. Inertial stability $I^{2}$ describes resistance of fluid elements to radial displacement, with $I^{2}>0$ representing inertially stable conditions. The inertial stability is proportional to the radial differential of squared absolute angular momentum per unit mass $M_{a}$, which is a function of $v, r$, and Coriolis parameter at the vortex center $f_{0}$ :

$$
\begin{aligned}
& M_{a}=r v+\frac{f_{0} r^{2}}{2} \\
& I^{2}=\frac{1}{r^{3}} \frac{\partial M_{a}^{2}}{\partial r}=\frac{1}{r^{2}}\left(r^{2} f_{0}^{2}+3 r v f_{0}+2 v^{2}\right)+\frac{1}{r} \frac{\partial v}{\partial r}\left(r f_{0}+2 v\right)
\end{aligned}
$$

Assuming all else equal, a vortex with a sharp decrease of $v$ along the radial direction will yield a smaller radial differential of $M_{a}$ (and thus $I^{2}$ ) than a vortex with a gradual decline of $v$. However, our composite framework precludes direct interpretation of $I^{2}$ due to the use of normalized radial space, and an exact calculation of any radial derivative using a discrete set of soundings is not possible. Variable intensity across composite space further complicates calculations of $I^{2}$.

To consider the variability of $V_{\max }$ in composite space, Fig. 9 shows the difference between estimated storm intensity and $v\left(\delta v=V_{\max }-v\right)$. A multiplicative normalization of $v$ was also examined $\left(v_{\star}=v / V_{\max }\right.$, not shown), but interpreting the radial variation of $v$ with a nondimensional field was a complicating factor (the findings from an analysis of $v_{\star}$ was similar to that of $\delta v$ ). In representing the departure of $v$ from the estimated $V_{\max }$ located near $r_{\star}=1$, we can interpret the spatial variation of $v$ with less obfuscation from sampling biases. As a proxy for $v$ in a vortex, a sharp increase of $\delta v$
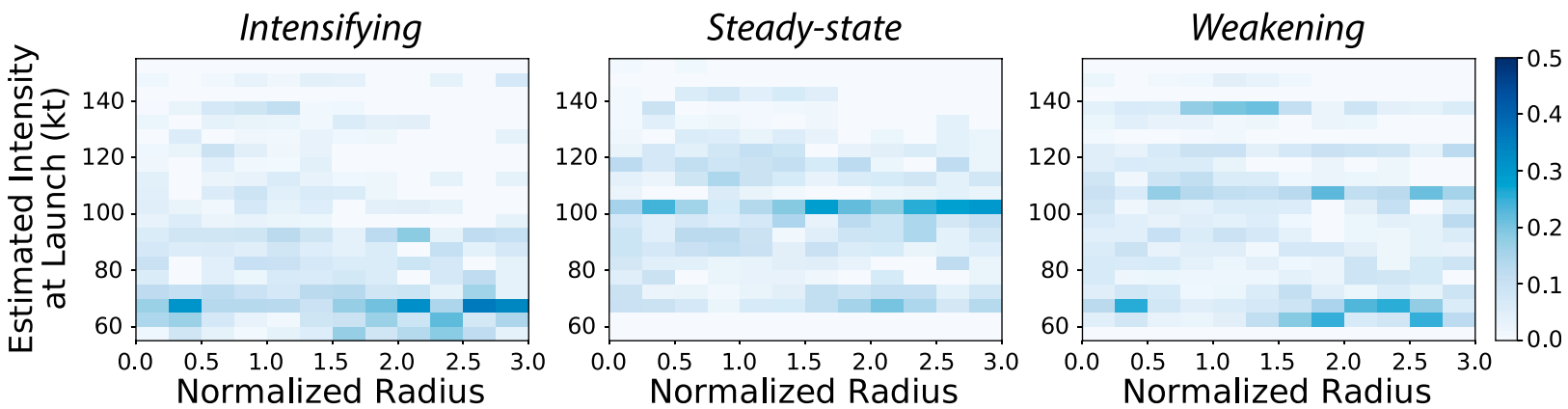

FIG. 5. Relative frequency of at-dropsonde-launch (hereafter "at-launch") estimated storm intensity (5-kt bin width) for each radial bin, expressed as a fraction. The value of a shaded cell (at $r_{\star}, V_{\max }$ ) represents the fraction of dropsondes launched at $r_{\star}$ with storm intensity $V_{\max }$ to the total number of launches at $r_{\star}$. Thus, the sum of all values in a column is unity. Variability across $r_{\star}$ signifies radial heterogeneity in sampling for a given $V_{\max }$, whereas variability across $V_{\max }$ implies diverse sampling of storm intensity for a given $r_{\star}$. 

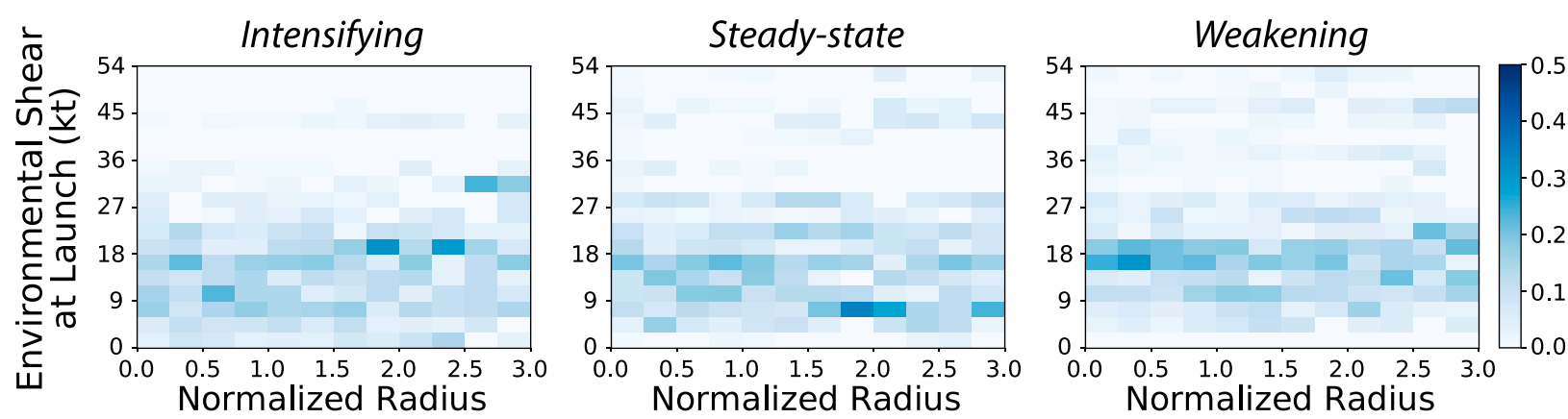

FIG. 6. As in Fig. 5, but the $y$ axis represents the at-launch environmental vertical wind shear magnitude (as estimated with SHIPS data).

The bin width used for shear data is $3 \mathrm{kt}$.

with radius implies a smaller radial differential of $M_{a}$, and thus weaker $I^{2}$.

Between $250-1500 \mathrm{~m}$, tangential winds in the IN group at $r_{\star}=(0.75,1.75]$ are mostly within $3 \mathrm{~m} \mathrm{~s}^{-1}$ of $V_{\max }$, suggesting a weak radial gradient of $v$ adjacent to and just outside of the RMW. Nonintensifying groups show a stronger gradient of $\delta v$ across $r_{\star}$ in the same region. Note that the differences of $\delta v$ between groups are generally due to data in nonintensifying storms being associated with larger RMW. The radial space where $\delta v<3 \mathrm{~m} \mathrm{~s}^{-1}$ in the SS group is closer to that seen in the IN group $-\delta v$ is less than $3 \mathrm{~m} \mathrm{~s}^{-1}$ between the RMW and $\sim 20-25 \mathrm{~km}$ outward from the RMW in IN and SS composites, compared to the WE composite where $\delta v<3 \mathrm{~m} \mathrm{~s}^{-1}$ spans between the RMW and $\sim 10-15 \mathrm{~km}$ outward from the RMW. In addition, the $\delta v$ "jet" is deepest in the IN group and more shallow in nonintensifying groups, which may be a consequence of stronger vertical mixing or turbulence in this region during intensification. These findings imply relatively high $I^{2}$ in this region during intensification compared to nonintensifying cases.

At greater radii $\left(r_{\star}>1.75\right)$ in $\mathrm{WE}, \delta v$ increases less with radius compared to other groups. The radial gradient of $\delta v$ at $r_{\star}>1.75$ should be smallest in the WE group and largest in the IN group, because the physical radii in this area are larger in nonintensifying storms (due to a larger RMW). These patterns suggest higher $I^{2}$

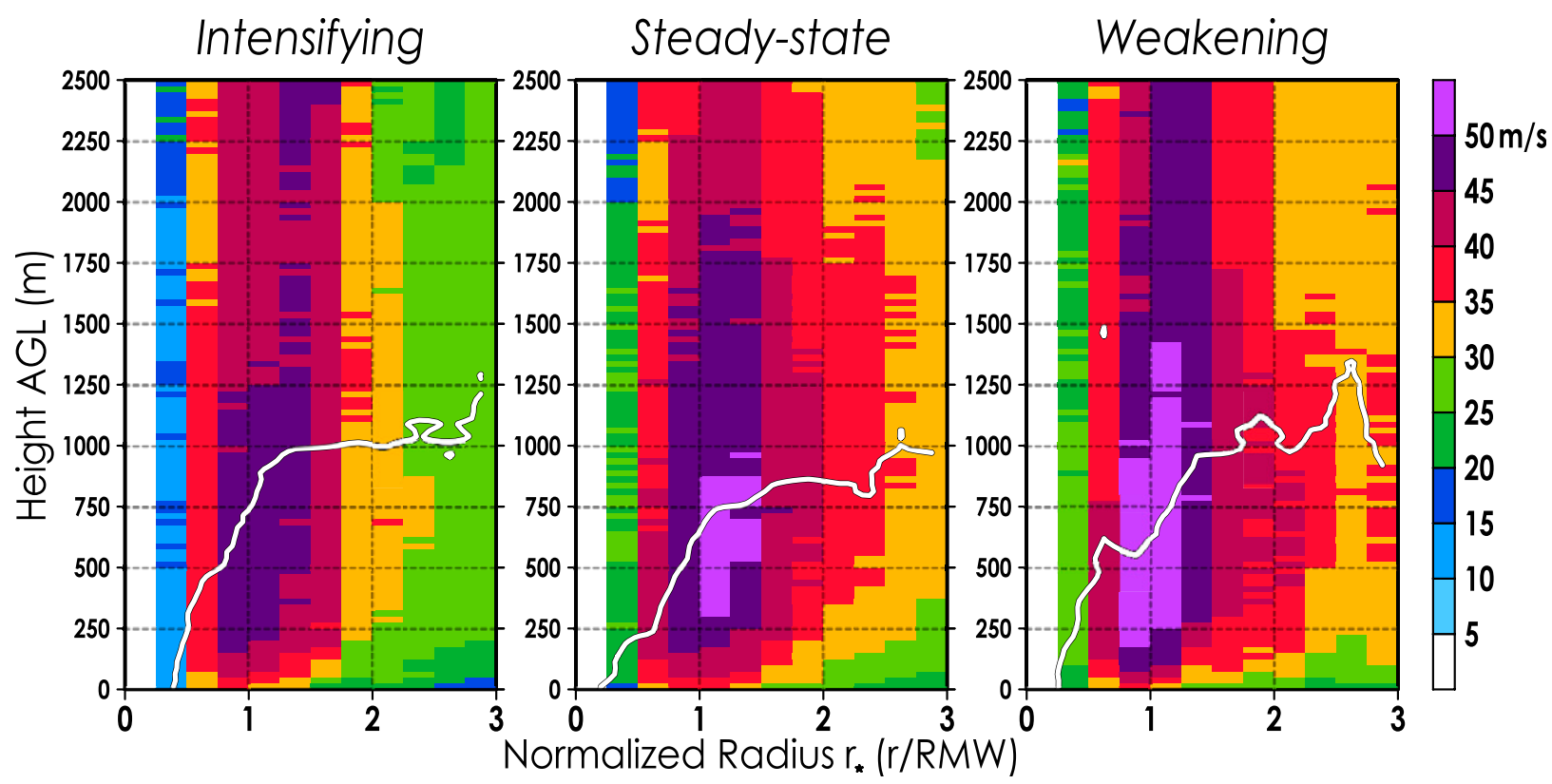

FIG. 7. Normalized radius-height cross sections of axisymmetric, storm-relative tangential velocity $v$ (in $\mathrm{m} \mathrm{s}^{-1}$ ) for all composite groups. The solid, white contour highlights the radial inflow layer adjacent to the surface (see Fig. 11). For defining the inflow layer, storm-relative radial velocity is smoothed 5 times with a 1-2-1 filter and then normalized by peak inflow. The inflow layer is where the normalized radial velocity is at least $10 \%$ of the peak inflow (omitted above $z=1.5 \mathrm{~km}$ ). 


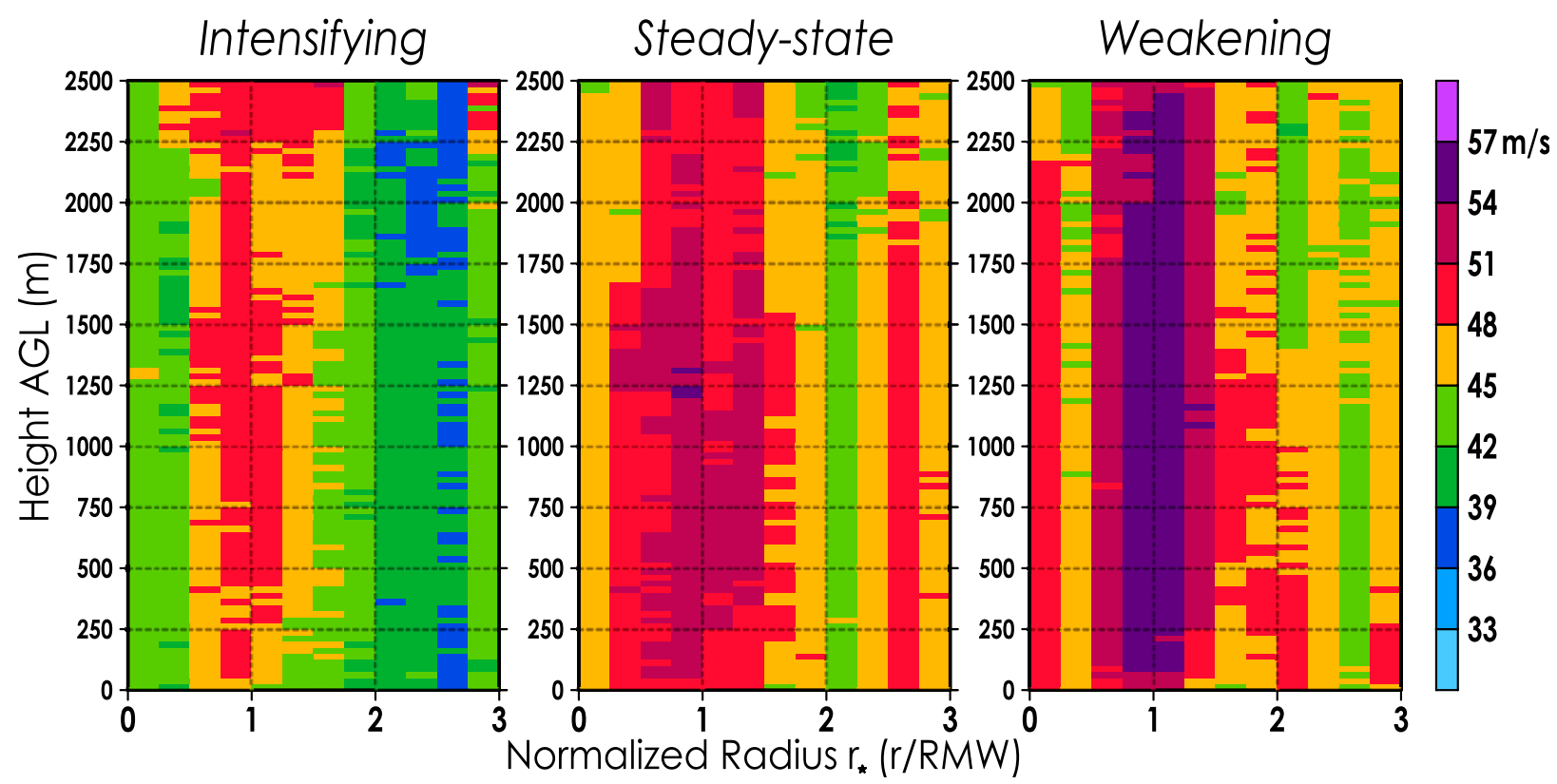

FIG. 8. Azimuthally averaged, normalized radius-height cross sections of estimated storm intensity, $V_{\max }$ (in $\mathrm{m} \mathrm{s}^{-1}$ ), at the time of sampling, interpolated from HURDAT2 data. Values of $V_{\max }$ associated with all samples in a given $r_{\star}-z$ bin are averaged to yield this figure.

in WE at these radii near the top of the inflow layer, and lower $I^{2}$ during intensification. Radially translating parcels that encounter higher $I^{2}$ in this region should have a greater tendency to be deflected vertically; this could enhance transport between the moist, near-surface inflow layer and the relatively dry air aloft. With the aid of radial convergence near the surface, shallow ascent of high-enthalpy air out of the $\mathrm{BL}$ is possible, whereupon local conditional stability can be reduced. These processes may explain observed

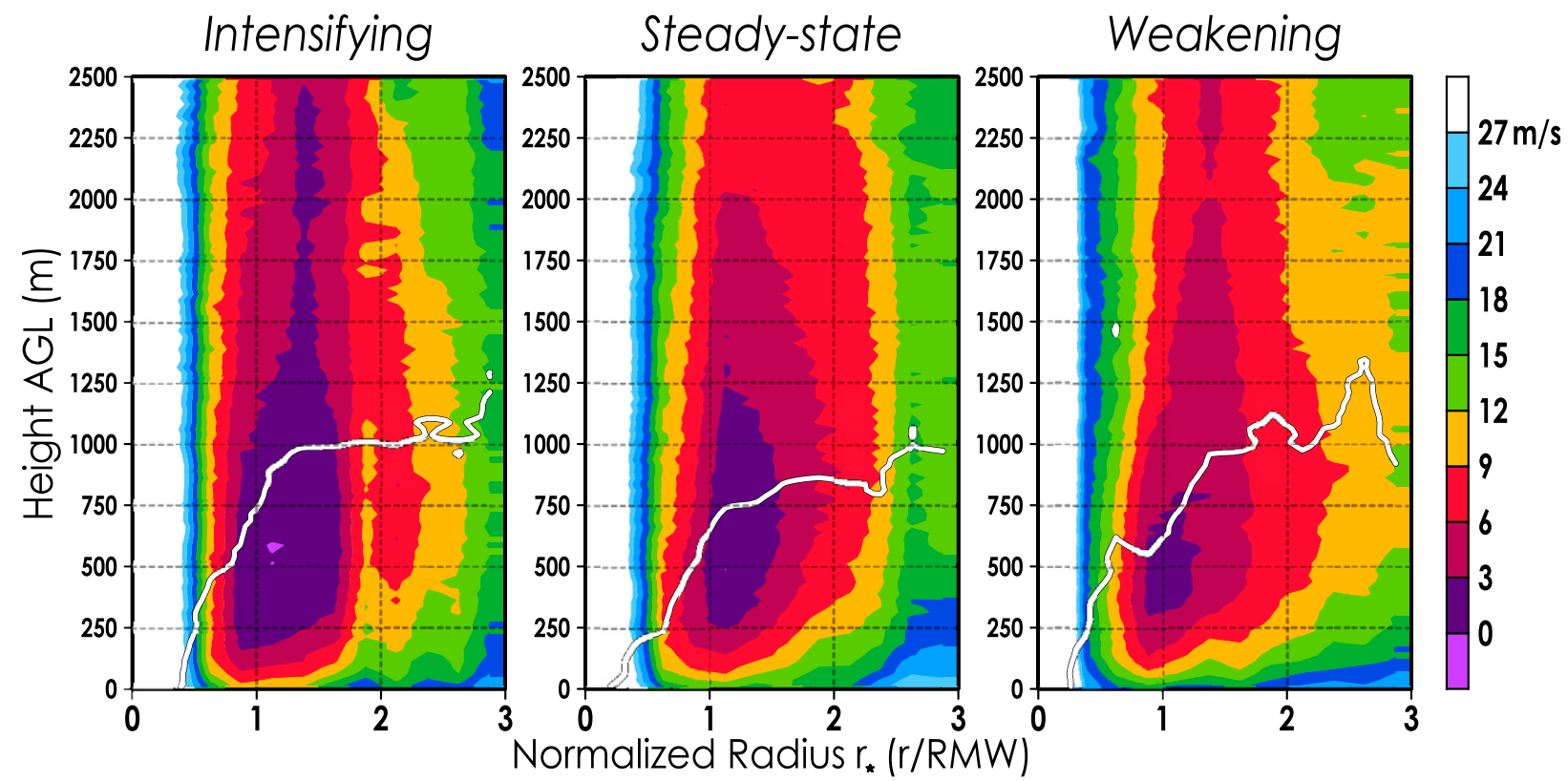

FIG. 9. Azimuthally averaged, normalized radius-height cross sections of the difference between estimated storm intensity and stormrelative tangential velocity, $\delta v=V_{\max }-v\left(\right.$ in $\mathrm{m} \mathrm{s}^{-1}$ ). The white contour marks the inflow layer height as defined in Fig. 11. 

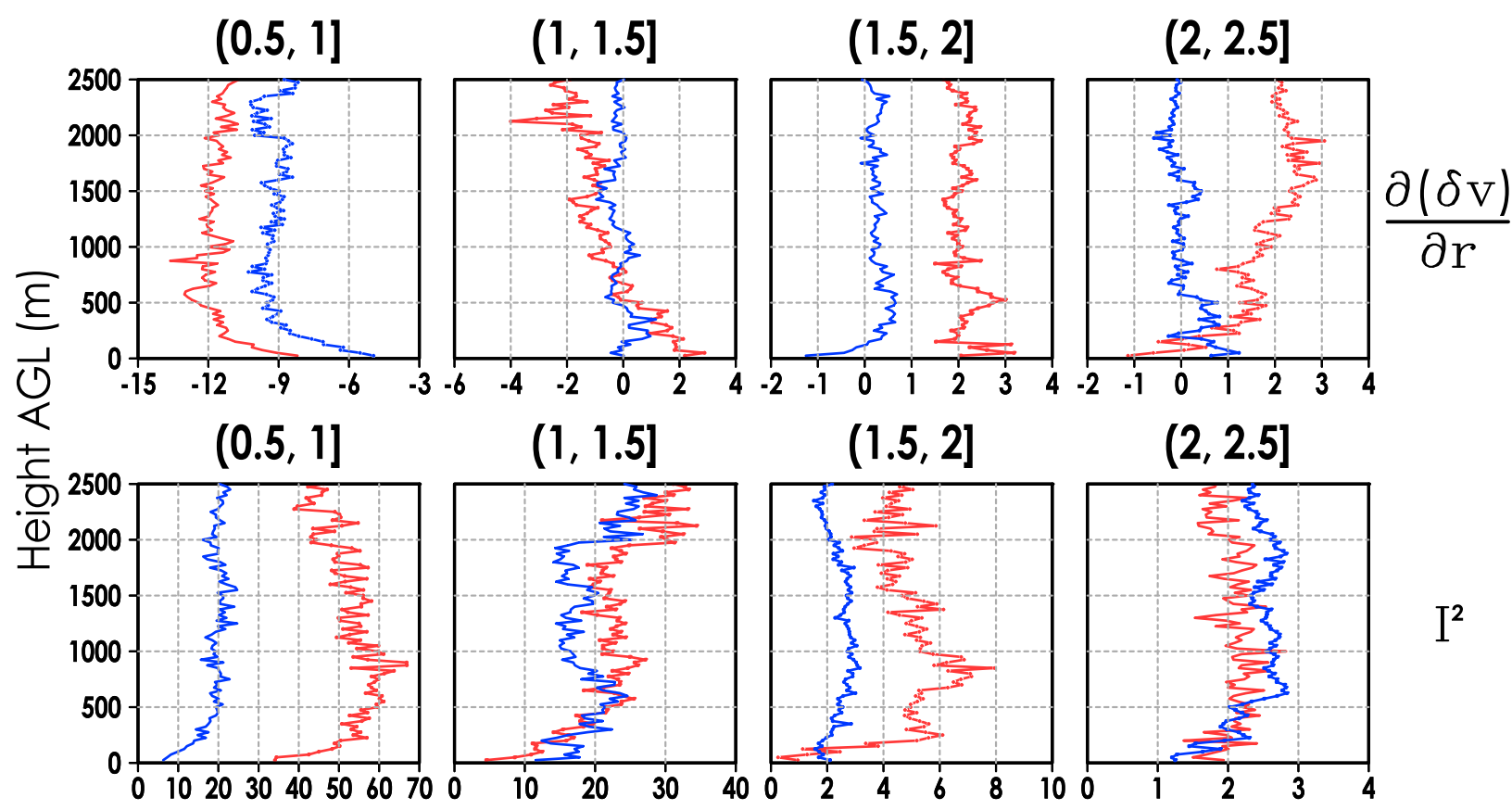

FIG. 10. Vertical profiles of (top) $\partial(\delta v) / \partial r$ (in $10^{-4} \mathrm{~s}^{-1}$ ) and (bottom) inertial stability $I^{2}$ (in $10^{-7} \mathrm{~kg}^{-2} \mathrm{~s}^{-2}$ ) using azimuthally averaged dropsonde data from Hurricane Earl (2010) during intensification (red profiles) and weakening (blue profiles). The top of each plot is labeled with the interval of $r_{\star}$ displayed. Both calculations used bin-averaged values for $r, v$, and $M_{a}$, with a 0.5 RMW radial resolution.

differences in radial location of deep convection between intensifying and nonintensifying storms from R13a, which also hypothesized that maximum BL convergence can be situated outside the RMW due to locally high $I^{2}$. Assuming low-level inertial stability is linked to $\mathrm{BL}$ convergence and deep convection, this analysis of $\delta v$ implies more (less) BL convergence and convection at the RMW for IN storms compared to nonintensifying storms, and more BL convergence and convection outside the RMW for WE storms.

This interpretation of composite $I^{2}$ rests on the assumption that an inverse relationship exists between $\partial(\delta v) / \partial r$ and $I^{2}$. An example of this relationship is found using bin-averaged dropsonde data from the wellsampled Hurricane Earl (2010) during intensification and weakening, shown in Fig. 10. With the exception of data from the $r_{\star}=(1.5,2]$ interval, $\partial(\delta v) / \partial r$ appears to have a nonlinear inverse relationship with $I^{2}$, as hypothesized. Earl's inertial stability is shown to be greatest during intensification inside the RMW, in agreement with the $\delta v$ field. Above $z=250 \mathrm{~m}, I^{2}$ tends to be weakest during intensification at $r_{\star}=(2,2.5]$. The breakdown of the relationship between $\delta v$ and $I^{2}$ apparent at $r_{\star}=(1.5,2]$ is due to a large radial gradient of sampled RMW in this range during Earl's weakening; the RMW increases from about $45 \mathrm{~km}$ at $r_{\star}=1.25$ to over $60 \mathrm{~km}$ at $r_{\star}>1.75$, which is nearly twice as large as the RMW found during Earl's intensification. With the strong dependence of $I^{2}$ on $r^{-1}$, the calculated $I^{2}$ for Earl at $r_{\star}=(1.5,2]$ during weakening is considerably lower than $I^{2}$ during intensification due to the RMW sampling bias. Note that this relationship breakdown due to sampling bias suggests that a bulk calculation of $I^{2}$ is sensitive to sampling biases (particularly in $r$ ), but does not necessarily suggest that $\delta v$ is a better approximator of inertial stability for biased data.

\section{b. Secondary circulation}

Normalized radial velocity $u_{\star}=u /\left|u_{\min }\right|$ is depicted in Fig. 11, which presents a surface-adjacent inflow layer in each composite. Relatively weak radial winds are seen throughout much of the region outside of the inflow layer. Near-surface inflow extends to $r_{\star}=0.5$, and deepens with increasing radius inside the RMW. The height of maximum wind speed generally follows near the top of the inflow, below which the effects of friction lead to momentum loss and substantial agradient forcing (Ooyama 1969; Smith et al. 2009).

Outside of the RMW, the inflow layer is roughly 750 $1250 \mathrm{~m}$ thick. At $r_{\star}=(1.0,1.5]$, the IN inflow layer is of greater or nearly equal thickness compared to the SS and WE groups, despite the greater intensity of storms in the nonintensifying groups (Figs. 5 and 8). This implies that the vertically integrated radial convergence at and immediately inward of the RMW is relatively high 


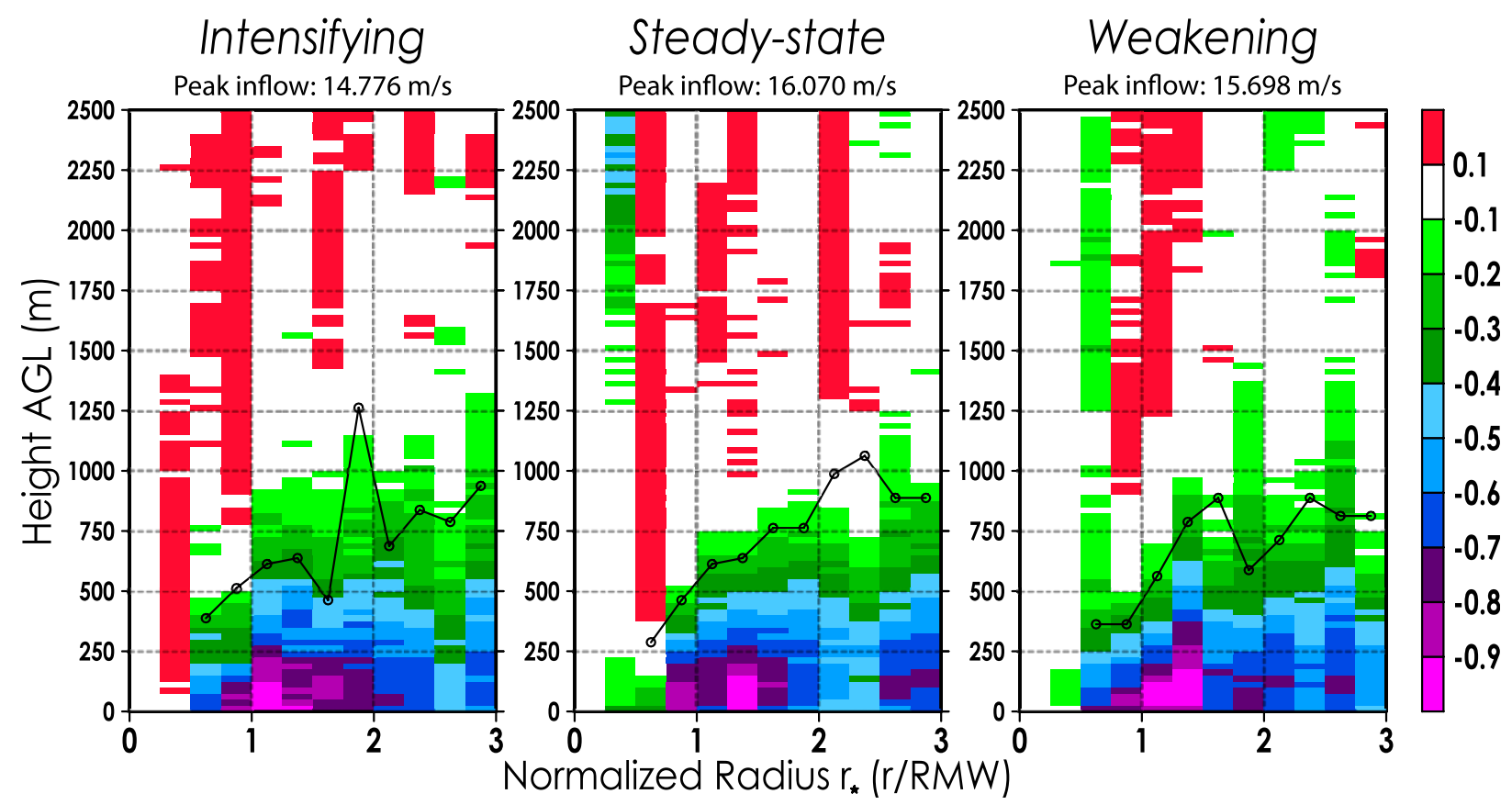

FIG. 11. Normalized radius-height cross sections of azimuthally averaged, storm-relative radial velocity normalized by the magnitude of peak inflow in each group (listed at the top of each panel). Negative values indicate inflow. Black lines connect black circles representing the height at which $v$ (smoothed with a 1-2-1 filter 5 times) is maximized at each radial bin outside of $r_{\star}=0.5$.

during intensification for given storm intensity. At $r_{\star}=$ $(1.25,2.75]$, the inflow layers of the IN and WE groups are often near $1000 \mathrm{~m}$ thick. If the depth of the frictional inflow layer scales with the ratio of turbulent diffusivity $K$ to inertial stability [specifically, $\sqrt{2 K / I}$, discussed in Kepert (2001); Kepert and Wang (2001)], then vertical diffusion in the WE group should be greater than IN at $r_{\star}=(1.25,2.75]$ (to account for enhanced $I^{2}$ implied in Fig. 9).

All composites show near-surface inflow maxima just outside of the RMW. Inflow weakens rapidly with decreasing radius inside the RMW, in tandem with high $I^{2}$ near the RMW. The radial extent of $u_{\star}<-0.7$ is most pronounced in nonintensifying storms. Strong radial inflow at multiple radii outside of the RMW may be indicative of tendencies for radial convergence at these radii. For instance, a local maximum of inflow exists below $250 \mathrm{~m}$ at $r_{\star}>2.5$ in the SS and WE groups, which could be a sign of enhanced convergence there relative to IN. However, note that sampling biases in intensity (Figs. 5 and 8) could translate to $u$ fields, because BL inflow is driven via agradient forcing that is dependent on surface friction and momentum aloft.

Prior work has suggested that diabatic heating located inside the RMW as a result of convection is favorable for intensification (Shapiro and Willoughby 1982; Pendergrass and Willoughby 2009; Vigh and Schubert 2009). By contrast, diabatic heating located radially outward of the RMW reduces the temperature gradient between the hurricane eye and the position of heating, thereby reducing the radial surface pressure gradient between these points and affecting the wind field. Based on our profiles of $u$ and $\delta v$ that imply more radial convergence and high $I^{2}$ at radii outside the RMW in the WE composite relative to IN and SS groups, storm weakening may be anticipated on the premise that convection is more likely outside of the eyewall [as seen in simulations by Hazelton et al. (2017a,b), for example], thus distributing diabatic heating over a larger area and reducing the core's temperature anomaly relative to the outer vortex. Similar arguments can be made considering advection of $M_{a}$ : Ascent out of the BL primarily inside the RMW (as implied for IN) would lead to near-surface inflow that advects relatively high- $M_{a}$ surfaces toward the RMW, which would spin up the primary circulation (Smith and Montgomery 2016). On the other hand, ascent out of the BL outside of the RMW (as implied for WE) would spin down the maximum tangential winds, as relatively low- $M_{a}$ surfaces would be advected outward into the RMW.

If radial convergence and forced shallow ascent are occurring outside the RMW (possibly due to higher $I^{2}$ outside the RMW as discussed earlier), it is possible that ascending air exits the BL and-pending its enthalpycould continue to ascend in convection. This event would come at the cost of the inflow's moist static 


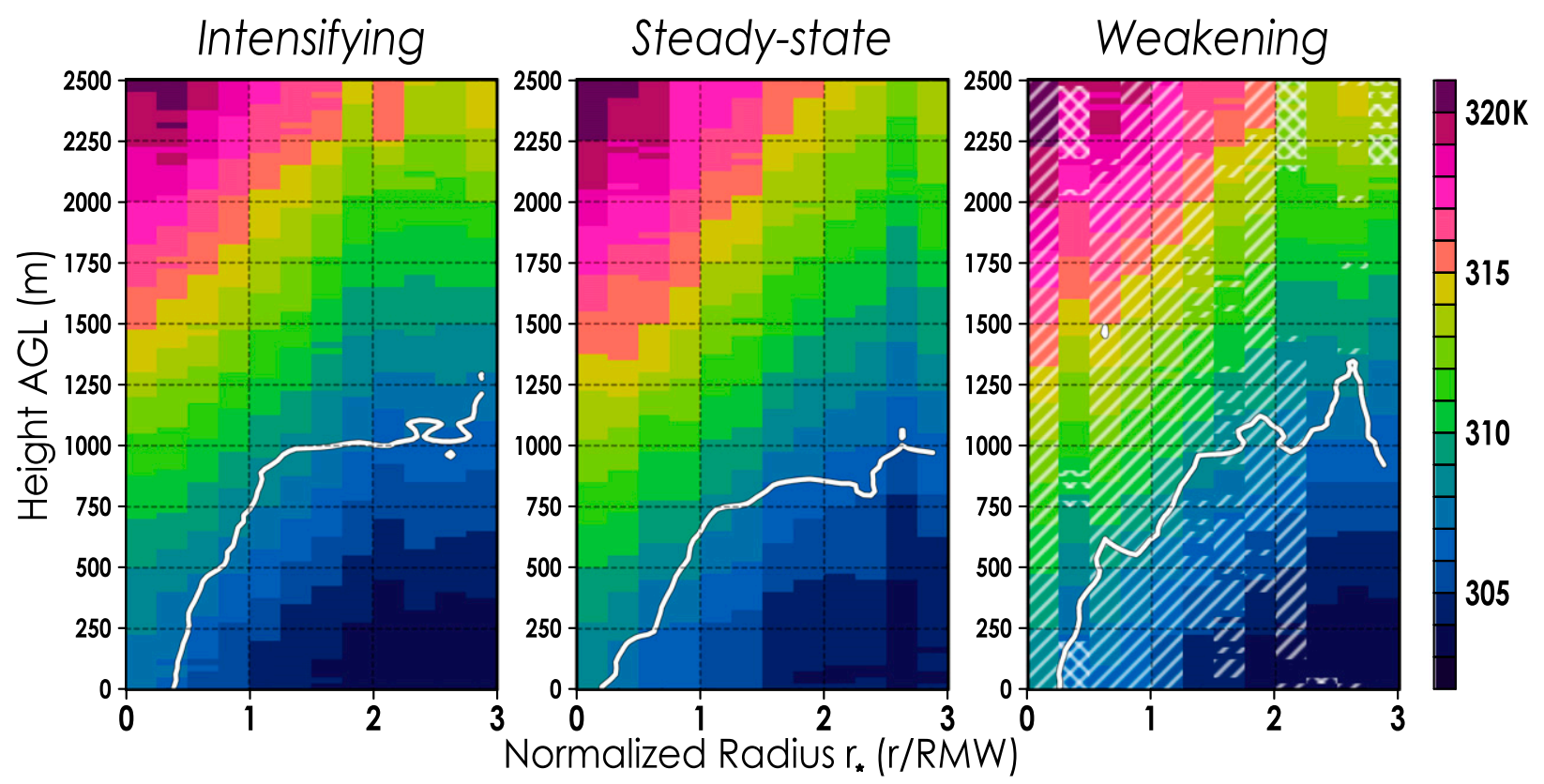

FIG. 12. As in Fig. 7, but virtual potential temperature $\theta_{v}$ (in K) is shaded. Differences between IN and WE with statistical significance (to $95 \%$ confidence) are depicted with diagonal lines (where $\theta_{v}$ is greater in WE) or cross hatching (where $\theta_{v}$ is greater in IN) in the WE panel.

energy, which would be reduced when high- $\theta_{e}$ parcels exit the inflow. Forced subsidence inward of such convergence transports relatively dry air from above the $\mathrm{BL}$ downward into the inflow, which should reduce the layer's enthalpy. Thus, it might be expected that BL $\theta_{e}$ outside of the RMW is low in the WE group compared to IN, due to implied differences in radial convergence outside the RMW. Convective downdrafts and turbulent mixing at these radii could also transport low-enthalpy air from the free atmosphere into the inflow layer below, which are not examined here explicitly. These processes can reduce the $\theta_{e}$ of inwardly moving air that arrives at the eyewall, affecting conditional stability and diabatic heating within the high $-I^{2}$ core. We will next examine these ideas in more detail.

\section{c. Thermodynamic structure}

Virtual potential temperature $\left(\theta_{v}\right)$ is plotted in Fig. 12, revealing a low-level, virtually warm-core structure typical of TCs in each composite. Within the RMW, nonintensifying storms are associated with warmer $\theta_{v}$ (up to about $2 \mathrm{~K}$ ) than intensifying storms. At $r_{\star} \leq 2, \theta_{v}$ in the IN group is often significantly lower than $\theta_{v}$ in the WE group. Knowing that the nonintensifying groups are comprised of more-intense hurricanes than the IN group on average, this result is sensible and corroborates findings by $\mathrm{Z} 11$.

Since our composite spaces' vertical coordinate is height, we can deduce static stability—-the Brunt-Väisälä frequency, displayed in Fig. 13-using $\theta_{v}$. Static stability is defined and calculated here as $N^{2}$ :

$$
N^{2}=\frac{g}{\theta_{v}} \frac{\partial \theta_{v}}{\partial z} .
$$

For calculating $N^{2}$, the vertical grid spacing of the composite data is increased to $100 \mathrm{~m}$, so that noise is reduced when using a centered finite difference to determine $\partial \theta_{v} / \partial z$.

Weaker static stability (i.e., more unstable air) is present within a layer at the surface. This layer of low $N^{2}$ is thickest at $r_{\star}>2$ in all groups, becoming shallower with decreasing radius toward the RMW. A very shallow layer of near-superadiabatic conditions is detected in each composite near $r_{\star}=2.5$, around $150 \mathrm{~m}$ AGL. In the lowest $750 \mathrm{~m}, N^{2}$ is largest inside the RMW in all cases. In the eye below $400 \mathrm{~m}$ and radially inward of the inflow layer, a region of weaker static stability is present in all groups-the least stable in IN, and the most stable in WE.

More statically stable conditions are seen above and about the top of the frictional inflow layer. Dry stability near the top of the inflow layer has been previously analyzed in observations and models (Z11; Z13; Kepert et al. 2016). Kepert et al. (2016) suggests this dry stability is a result of diabatic cooling from evaporating rainfall; and differential temperature advection via radial flow, with cold-air advection decreasing with height. Above the inflow layer at $r_{\star}>2$, nonintensifying groups have 


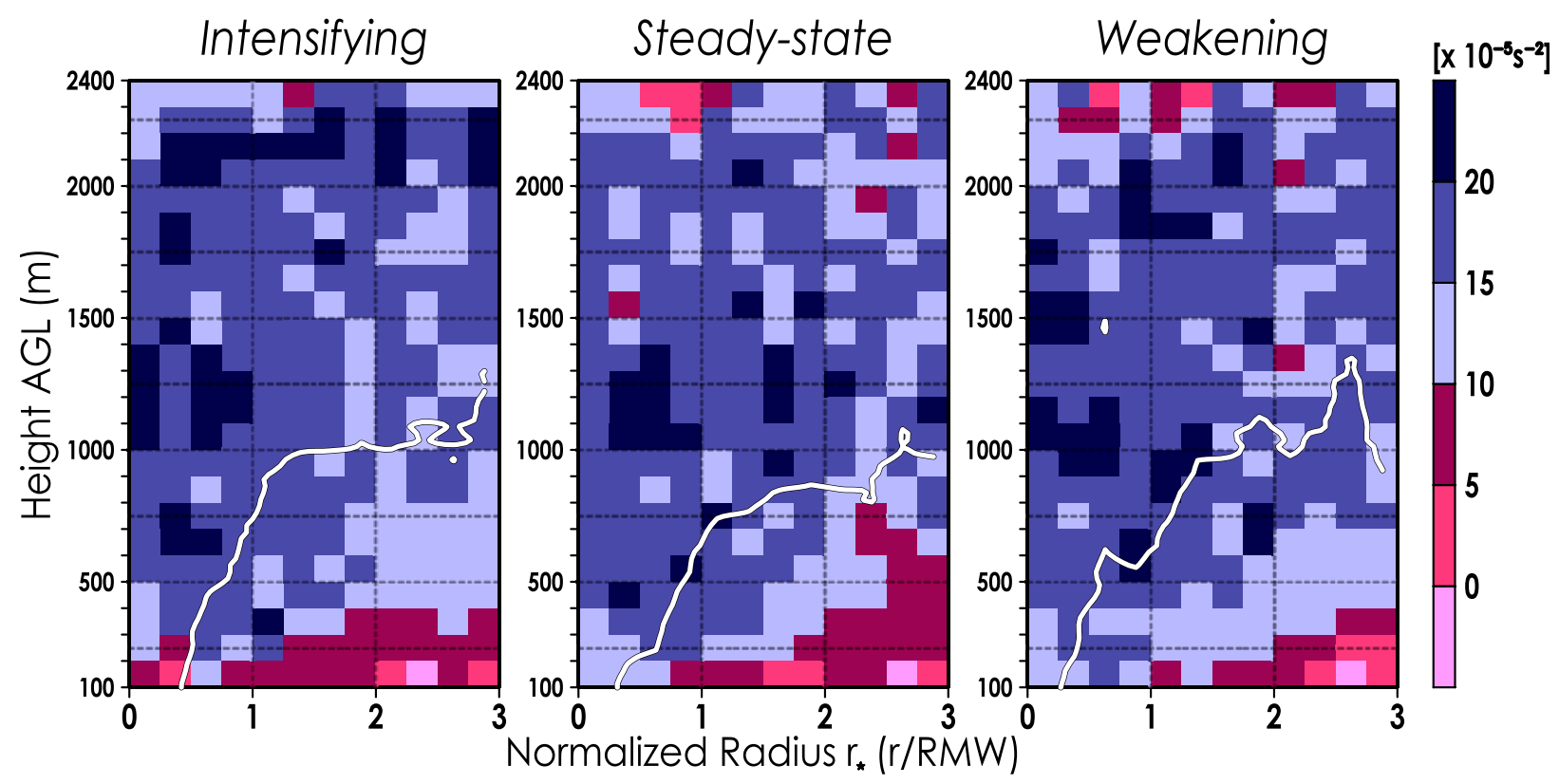

FIG. 13. Azimuthally averaged, normalized radius-height cross sections of static stability, $N^{2}$ (in $10^{-5} \mathrm{~s}^{-2}$ ), plotted between 100 and $2400 \mathrm{~m}$ above ground level. The data in this figure were composited into lower-resolution vertical bins with 100-m thickness to reduce noise. The vertical derivative of $\theta_{v}$ is calculated using a centered finite difference. The white line is defined identically as in Fig. 7.

generally lower $N^{2}$ than the IN group. If relatively deep vertical oscillations are more frequent in this region for nonintensifying storms, then thermal and kinematic properties above the top of the TCBL may have greater potential to entrain with near-surface inflow at these radii during SS and WE. In this case, we might expect the entropy of inflowing parcels to be reduced before arriving at the eyewall, provided they are not removed from the inflow layer via frictional ascent and/or convection.

Axisymmetric cross sections of $\theta_{e}$ are shown in Fig. 14. In each group, a core of high- $\theta_{e}$ air is evident inside the RMW. This feature has been seen before (Bell and Montgomery 2008), but there are differences between the composites. Inside the RMW, the IN group has the lowest $\theta_{e}$ (mostly between 357 and $363 \mathrm{~K}$ ) and most-negative $\partial \theta_{e} /$ $\partial z$. The nonintensifying groups have greater and more vertically homogeneous $\theta_{e}$ in this region - often between 360 and $366 \mathrm{~K}$. Above $z=1 \mathrm{~km}$ inside the RMW, $\theta_{e}$ in WE is generally greater than that of IN to a statistically significant degree. An oft-negative vertical gradient of $\theta_{e}$ outside of the RMW is seen in all groups below $z \approx 500 \mathrm{~m}$ [similar to Barnes (2008)].

At $r_{\star} \leq 0.5$, we see the greatest values of $\theta_{e}$ in the domain for all groups adjacent to the surface. Parcels that translate radially into this region should interact and mix with the high-entropy air in the eye, as there is a gradient of $\theta_{e}$ across $r_{\star}=0.5$. Parcels that enter the eye eventually exit in some fashion, and they may transport additional energy acquired from the low-level eye. The degree to which a parcel's $\theta_{e}$ has mixed with the limited reservoir of high-entropy air is dependent on the parcel's time spent there, the amount of turbulence in the low-level eye, and the $\theta_{e}$ difference between the parcel and its surroundings (Cram et al. 2007; Bell and Montgomery 2008; Guimond et al. 2016; Hazelton et al. 2017a,b).

In the IN group, a region of $\theta_{e} \gtrsim 366 \mathrm{~K}$ is isolated below $z \approx 250 \mathrm{~m}$ and adjacent to the innermost boundary of the inflow layer. Parcels that move into this high$\theta_{e}$ area should increase their enthalpy via entrainment, and then return to the eyewall region (Persing and Montgomery 2003; Hazelton et al. 2017a). The vertical gradient of $\theta_{e}$ for IN inside the RMW is most pronounced, so eye-penetrating parcels that mix with the inner eyewall after some time in the eye may be buoyant, ascend above the $\mathrm{BL}$, and release latent heat inside the high- $I^{2}$ core. Nonintensifying groups have a weaker $\partial \theta_{e} / \partial z$ inside the RMW. Parcels in the eye that mix with the inner eyewall in nonintensifying groups may encounter greater moist static stability unless they acquire more enthalpy. A thin layer of $\theta_{e}>369 \mathrm{~K}$ is adjacent to the surface in SS and WE composites, which allows a parcel to achieve higher $\theta_{e}$ than suggested in IN.

Relatively low $\theta_{e}$ extends deep into the WE composite's inflow layer at $r_{\star}>2.25$ (to about $250 \mathrm{~m}$ in the farthest radial bin). The difference in $\theta_{e}$ between IN and WE groups is statistically significant in some of this 


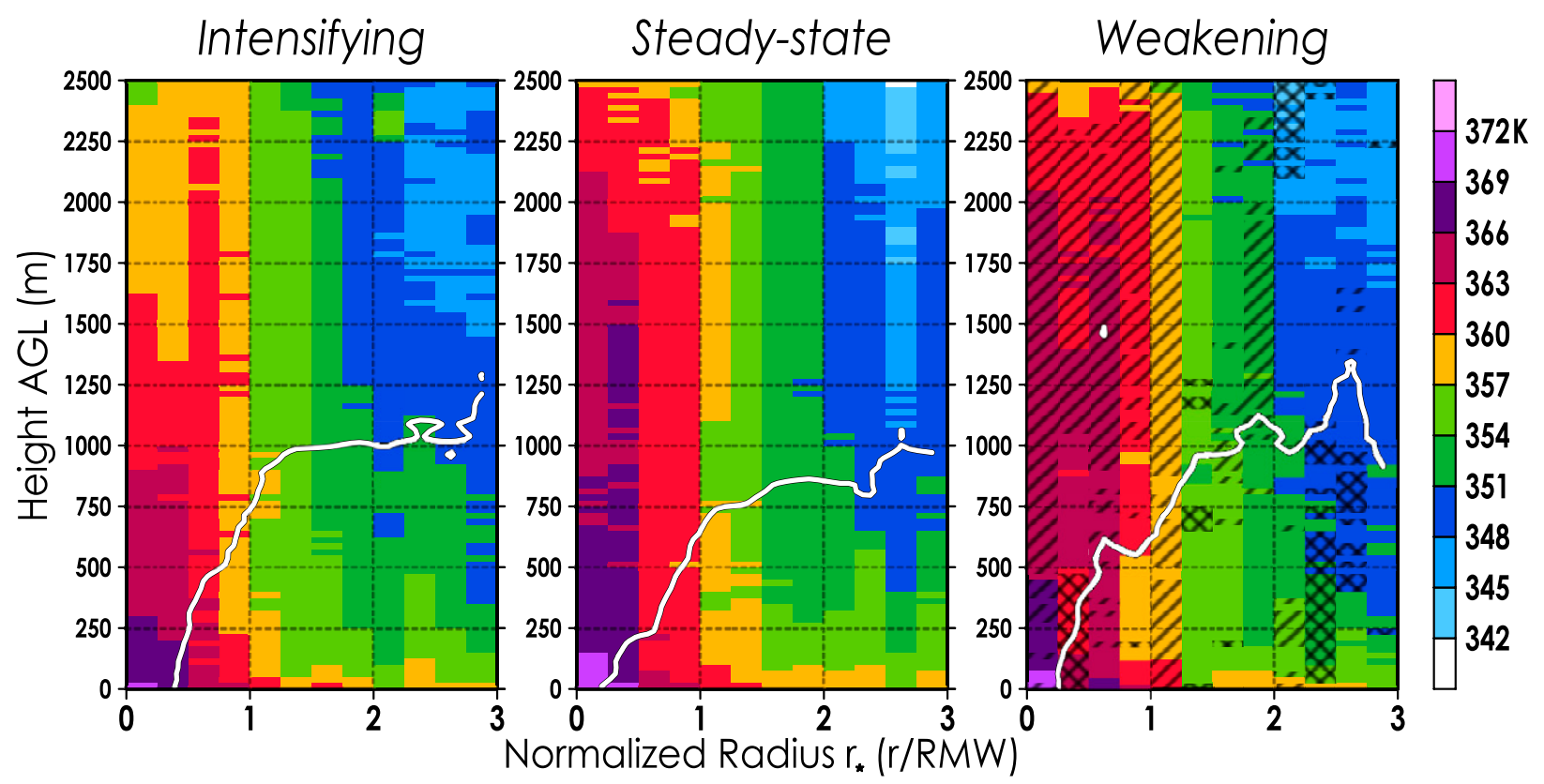

FIG. 14. As in Fig. 7, but equivalent potential temperature $\theta_{e}$ (in $\mathrm{K}$ ) is shaded. Statistically significant differences between IN and WE are plotted the same as in Fig. 12 (using a darker color for clarity).

region. This difference could be explained by an increased frequency or strength of convective downdrafts extending downward into the BL locally or at greater radii (whereby lower $\theta_{e}$ could be advected inward). The lower $\theta_{e}$ could also be a signal of forced subsidence into the BL due to convergence and shallow ascent out of the BL at smaller radii (Ooyama 1969; Kepert 2013), or increased turbulent mixing in the presence of vertical shear (Kepert 2010). By the aforementioned processes, low- $\theta_{e}$ air may entrain with the strong near-surface inflow. Parcels from outer radii may increase their $\theta_{e}$ before arriving at the RMW (and possibly overshooting into the eye), depending on the parcels' distance traveled, translational speed, further entrainment from outside the inflow, and $\mathrm{OHC}$.

Just inside the RMW in WE, there are signs of conditional stability where $\theta_{e}$ increases with height. This same region in the SS group has higher $\theta_{e}$ despite being associated with weaker hurricanes, which implies some difference in the thermal properties of the secondary circulation. Lower $-\theta_{e}$ parcels entering the eye in WE may have to acquire more enthalpy than the IN or SS groups before returning to the eyewall if they are to be buoyant inside the RMW. Otherwise, upon returning to the eyewall, these parcels would not be buoyant upon reaching saturation unless they move outward to an environment with lower $\theta_{e}$, implying more-slantwise eyewall convection in WE. If parcels move outside of the RMW and become buoyant in WE, any diabatic heating associated with ascent of these parcels will reduce the temperature gradient across the RMW, which through thermal wind arguments should result in an adjustment of the primary circulation toward weaker winds below the reduced temperature gradient. On the other hand, if these parcels are forced to ascend at or within the RMW-for instance, because of ongoing convection-and are conditionally stable, then saturation will not prevent these parcels from decelerating preexisting vertical motion in the eyewall.

The SS group exhibits nearly moist-neutral conditions between $r_{\star}=0.5$ and the RMW up to $z \approx 2 \mathrm{~km}$. Parcels entering the eye from the inflow layer originate from an almost conditionally neutral or slightly stable environment. A deep layer of $\theta_{e}>366 \mathrm{~K}$ is seen in the low-level eye, indicating a larger volume of high-entropy air with which overshooting parcels can interact. Air moving into the eye may be more able to increase its enthalpy than the other groups, but it is difficult to discern (from this data, at least) how much these parcels will interact with the column of higher $\theta_{e}$ air above the surface. Regardless, the moist-neutral conditions above the inflow layer in the eyewall suggest that there are less parcels in the SS group mixing with the inner eyewall that could considerably accelerate or decelerate local vertical motion upon saturation.

\section{Summary and discussion}

To further our understanding of the tropical cyclone boundary layer (TCBL) and its role in hurricane growth, 
decay, and maintenance, sounding data from Atlantic dropsondes launched between 1998 and 2015 were gathered and sorted into composite datasets based on intensity change-groups of data from intensifying, steady-state, or weakening storms. From an original set of 12045 soundings gathered, 3091 were ultimately used for analysis. The datasets utilized a combination of SFMR for detecting RMW (Uhlhorn and Black 2003; Uhlhorn et al. 2007; Klotz and Uhlhorn 2014), HURDAT2 for indicating intensity and intensity change (Landsea and Franklin 2013), SHIPS for shear-relative positioning (DeMaria et al. 2005), and 2-min track data for TC location (Willoughby and Chelmow 1982).

The goal of this research was to identify kinematic and thermodynamic properties of the BL associated with intensifying and nonintensifying hurricanes, and then explain the relevance of such properties in the context of storm evolution. While this research is comparable to prior work examining the TCBL using dropsondes (F03; Bell and Montgomery 2008; Barnes 2008; Z11; Z13) and intensity change using airborne Doppler data (R13a), this analysis is unique in its investigation of axisymmetric TCBL structure as a function of intensity change using a more comprehensive observational dataset.

The composite datasets incorporate information from multiple hurricanes with varying properties (e.g., intensity, size, translational speed, structure). A climatology of the data revealed the storms sampled by dropsondes, the locations of dropsonde launches in Earth- and storm-relative spaces, and the spatial distributions of average storm intensity and environmental vertical wind shear in composite space. In our analysis, most sampling in the Caribbean Sea and southwestern Gulf of Mexico is associated with intensifying storms, which implies that the environment is more conducive for intensification [e.g., high OHC (Leipper and Volgenau 1972), weak environmental shear (DeMaria 1996; Gallina and Velden 2002; Paterson et al. 2005)]. A large amount of data originates elsewhere in the Gulf of Mexico and off the eastern U.S. coastline, where influences of nearby land, large-scale extratropical systems, and spatially variable SSTs may impede or promote intensification. Most dropsondes are launched in the eye and eyewall regions, and less sampling occurs outside of these areas.

Each composite revealed a low-level tangential wind jet below $1250 \mathrm{~m}$ AGL, and an almost logarithmic decrease of $v$ with decreasing height below the jet [in alignment with F03 and Powell et al. (2003)]. A stronger inner-core primary circulation is evident in WE, whereas IN is associated with weaker $v$. Intensity sampling in each composite showed that these differences across groups are at least partially due to sampling bias.
On average, weaker storms are sampled during intensification, and comparably intense storms are associated with weakening.

A difference field between intensity and $v$ was calculated to mitigate this sampling bias obscuring interpretation of the results. In this difference field, we found the IN composite was associated with the deepest jet in the eyewall region, which is indicative of stronger vertical turbulence or motion in the area compared to nonintensifying storms. The same difference field was also used to infer inertial stability. The eyewall region in IN appeared more inertially stable, and the region above the near-surface inflow outside of the eyewall was less stable compared to nonintensifying groups. These results suggest that nonintensifying hurricanes could be associated with an increased likelihood for low-level ascent outside of the RMW - at least compared to intensifying hurricanes. The deep jet of the IN composite, along with the implication of high $I^{2}$ in the eyewall and low $I^{2}$ outside the eyewall, indicates that convection could be mostly confined about the eyewall and/or near the RMW during intensification.

We followed with examinations of the secondary circulation in each composite. All composites presented a surface-adjacent inflow layer, largely situated beneath a local wind maximum. All groups exhibited strong, nearsurface inflow close to the RMW. The inflow extends through the RMW, suggesting eyewall penetration near the surface. Nonintensifying groups had a larger radial extent of substantial inflow outside the RMW, which we interpret as increased potential for BL convergence away from the RMW. By this logic, the nonintensifying composites are associated with ascent and possibly convection outside the RMW (at least more so than IN), which could weaken the TC. Caution should be taken with this last finding, however, as the intensity sampling bias could be partially responsible for composite differences in radial flow [for example, Z11 found an $8.5 \mathrm{~m} \mathrm{~s}^{-1}$ difference in composite peak inflow between category 1-3 storms and category 4-5 storms].

Thermodynamic properties of the hurricane BL were presented after the kinematics. Composites of nonintensifying hurricanes had the warmest $\theta_{v}$ inside the RMW, which is likely a reflection of average storm intensity. Within the frictional inflow layer in all composites, measures of $N^{2}$ were low compared to outside the inflow layer. More statically stable air overlays the inflow layer, which corroborates prior work (Z11; Kepert et al. 2016). Above the inflow layer and away from the RMW $\left(r_{\star}>2\right), N^{2}$ is generally reduced in nonintensifying composites compared to IN. This property suggests that vertical motions between the inflow layer and air aloft might be more prevalent in 
nonintensifying storms at these radii. If the air above the BL has less enthalpy than inflowing air, then vertical exchange between the BL and the free atmosphere would reduce moist static energy of the inflow approaching the eyewall.

We concluded with an examination of equivalent potential temperature $\theta_{e}$. All groups have high- $\theta_{e}$ eyes, with a generally negative vertical gradient of $\theta_{e}$ inside the RMW. The IN composite presented the most pronounced vertical gradient of $\theta_{e}$ in the eye. KelvinHelmholtz instabilities along a hurricane's inner eyewall can mix high-entropy air of the low-level eye into the eyewall, possibly introducing positive buoyancy and affecting convection (Montgomery et al. 2006). Conditional stability in the eye differed across groups, suggesting differing consequences to horizontal mixing between the eye and nearly moist-neutral eyewall. The most conditionally unstable eye was seen in IN, implying that conditional instability could be most readily introduced to the eyewall during intensification if horizontal mixing occurred through sufficient depth. Outside of the eyewall, relatively low $\theta_{e}$ extends into the near-surface inflow in all groups; however, this feature extends deepest into the BL in the WE composite. This low-entropy air from outside the BL can entrain with the frictional inflow most effectively in weakening storms, which we hypothesize can affect the air that eventually arrives at the eyewall. Possible explanations behind this difference in composites include lower $\mathrm{OHC}$ and reduced surface heat fluxes, vertical motions near the top of the BL (i.e., from downdrafts and/or frictionally forced ascent and descent), and increased vertical diffusion from vertical shear.

To summarize, several kinematic and thermodynamic differences are seen in the BL between intensifying and nonintensifying hurricanes using composited dropsondes. Similar to results from Z11, the thickness of the radial inflow layer in our composites decreases with decreasing radius in the inner core regardless of stratification by intensity change; and the height of maximum tangential wind is consistently near the top of, but within, the inflow layer. This study found that the structure of near-surface inflow differed between intensifying, steady-state, and weakening hurricanes in the Atlantic basin. This implies differences in BL convergence as a function of radius between states of intensity change, which is linked to frictional updrafts at the top of the BL and potentially convection. Specifically, the IN composite conveys a relatively strong profile of radial convergence inside the RMW (given the average intensity associated with sampled storms), while nonintensifying composites suggest stronger convergence at radii well outside of the RMW. Coincident with these results are implied differences in inertial stability outside of the RMW (higher $I^{2}$ in nonintensifying groups), as well as lower BL $\theta_{e}$ in the WE composite at $r_{\star}>2.25$ (potentially due to an increased frequency/magnitude of convective downdrafts, forced subsidence, or vertical mixing). Breaking down these differences in a shearrelative framework (similar to Z13) should be a topic of future research.

This composite analysis of the hurricane BL during intensity change leaves us with ideas of processes that might affect hurricane intensity or structure. The key questions we have going forward are:

- How important is the structure of the low-level primary circulation in determining regions of ascent out of the BL (e.g., via frictional convergence in the inflow layer and/or vertical deflection due to $I$ )?

- How strongly is frictional convergence in the BL related to deep convection outside of the eyewall?

- How variable is low-level dry static stability outside of the RMW, and is it an important factor in convection outside of the eyewall?

- How do moist thermodynamic properties of the lowlevel TC eye and near-surface inflow layer affect intensity?

These questions are challenging to address adequately in an observational analysis, and especially in a composite analysis. Structural asymmetries were not comprehensively discussed here, which might be more appropriate for a modeling or highly sampled case study [such as those by Zawislak et al. (2016) and Rogers et al. (2016), whose two-part observational study examined the 3D kinematic and thermodynamic structure of Hurricane Edouard (2014) during varying modes of intensity change]. As the amount of hurricane observations increases, an observational examination similar to this one with a focus on asymmetric structure could be undertaken.

Acknowledgments. The authors acknowledge and thank two anonymous reviewers for their constructive criticism and recommendations that clarified and reinforced the direction and findings of this manuscript. We are grateful for comments and advice from the scientists at the Hurricane Research Division (HRD), which helped refine this work. This study also necessitated a large amount of data provided generously by HRD. Kyle Ahern was funded by NASA/JPL Contract 1419699, NGI Award 191001-363513.01C (NOAA Prime NA16OAR4320199), and NOAA Award NA17OAR4310153. Jun Zhang was supported by NSF Grants AGS1822128 and AGS1654831, and NOAA Grant NA14NWS4680030. 


\section{REFERENCES}

Ahern, K., and L. Cowan, 2018: Minimizing common errors when projecting geospatial data onto a vortex-centered space. $G e$ ophys. Res. Lett., 45, 12 032-12 039, https://doi.org/10.1029/ 2018 GL079953.

Barnes, G. M., 2008: Atypical thermodynamic profiles in hurricanes. Mon. Wea. Rev., 136, 631-643, https://doi.org/10.1175/ 2007MWR2033.1.

Bell, M. M., and M. T. Montgomery, 2008: Observed structure, evolution, and potential intensity of category 5 Hurricane Isabel (2003) from 12 to 14 September. Mon. Wea. Rev., 136, 2023-2046, https://doi.org/10.1175/2007MWR1858.1.

Black, M., J. Gamache, F. Marks Jr., C. Samsury, and H. Willoughby, 2002: Eastern Pacific Hurricanes Jimena of 1991 and Olivia of 1994: The effect of vertical shear on structure and intensity. Mon. Wea. Rev., 130, 2291-2312, https://doi.org/10.1175/ 1520-0493(2002)130<2291:EPHJOA > 2.0.CO;2.

Black, P. G., and Coauthors, 2007: Air-sea exchange in hurricanes: Synthesis of observations from the Coupled Boundary Layer Air-Sea Transfer experiment. Bull. Amer. Meteor. Soc., 88, 357-374, https://doi.org/10.1175/BAMS-88-3-357.

Chen, S. S., J. A. Knaff, and F. D. Marks Jr., 2006: Effects of vertical wind shear and storm motion on tropical cyclone rainfal asymmetries deduced from TRMM. Mon. Wea. Rev., 134, 3190-3208, https://doi.org/10.1175/MWR3245.1.

, W. Zhao, M. A. Donelan, J. F. Price, and E. J. Walsh, 2007: The CBLAST-Hurricane Program and the next-generation fully coupled atmosphere-wave-ocean models for hurricane research and prediction. Bull. Amer. Meteor. Soc., 88, 311-317, https://doi.org/10.1175/BAMS-88-3-311.

Corbosiero, K. L., and J. Molinari, 2002: The effects of vertical wind shear on the distribution of convection in tropical cyclones. Mon. Wea. Rev., 130, 2110-2123, https://doi.org/10.1175/ 1520-0493(2002)130<2110:TEOVWS > 2.0.CO;2.

— , and _ 2003: The relationship between storm motion, vertical wind shear, and convective asymmetries in tropical cyclones. J. Atmos. Sci., 60, 366-376, https://doi.org/10.1175/ 1520-0469(2003)060<0366:TRBSMV > 2.0.CO;2.

Cram, T. A., J. Persing, M. T. Montgomery, and S. A. Braun, 2007: A Lagrangian trajectory view on transport and mixing processes between the eye, eyewall, and environment using a highresolution simulation of Hurricane Bonnie (1998). J. Atmos. Sci., 64, 1835-1856, https://doi.org/10.1175/JAS3921.1.

Dee, D. P., and Coauthors, 2011: The ERA-Interim reanalysis: Configuration and performance of the data assimilation system. Quart. J. Roy. Meteor. Soc., 137, 553-597, https://doi.org/ 10.1002/qj.828

DeHart, J. C., R. A. Houze Jr., and R. F. Rogers, 2014: Quadrant distribution of tropical cyclone inner-core kinematics in relation to environmental shear. J. Atmos. Sci., 71, 2713-2732, https://doi.org/10.1175/JAS-D-13-0298.1.

DeMaria, M., 1996: The effect of vertical shear on tropical cyclone intensity change. J. Atmos. Sci., 53, 2076-2088, https://doi.org/ 10.1175/1520-0469(1996)053<2076:TEOVSO > 2.0.CO;2.

_ - M. Mainelli, L. K. Shay, J. A. Knaff, and J. Kaplan, 2005: Further improvements to the Statistical Hurricane Intensity Prediction Scheme (SHIPS). Wea. Forecasting, 20, 531-543, https://doi.org/10.1175/WAF862.1.

Emanuel, K. A., 1986: An air-sea interaction theory for tropical cyclones. Part I: Steady-state maintenance. J. Atmos. Sci., $\mathbf{4 3}$ 585-605, https://doi.org/10.1175/1520-0469(1986)043<0585: AASITF $>2.0 . \mathrm{CO} ; 2$.
_ 1988: The maximum intensity of hurricanes. J. Atmos. Sci., 45, 1143-1155, https://doi.org/10.1175/1520-0469(1988)045<1143: $\mathrm{TMIOH}>2.0 . \mathrm{CO} ; 2$

- 1995: Sensitivity of tropical cyclones to surface exchange coefficients and a revised steady-state model incorporating eye dynamics. J. Atmos. Sci., 52, 3969-3976, https://doi.org/ 10.1175/1520-0469(1995)052<3969:SOTCTS $>2.0 . C O ; 2$.

Franklin, J. L., M. L. Black, and K. Valde, 2003: GPS dropwindsonde wind profiles in hurricanes and their operational implications. Wea. Forecasting, 18, 32-44, https://doi.org/10.1175/ 1520-0434(2003)018<0032:GDWPIH > 2.0.CO;2.

Gallina, G. M., and C. S. Velden, 2002: Environmental vertical wind shear and tropical cyclone intensity change utilizing enhanced satellite derived wind information. Extended $A b$ stracts, 25th Conf. on Hurricanes and Tropical Meteorology, San Diego, CA, Amer. Meteor. Soc., 172-173.

Guimond, S. R., G. M. Heymsfield, P. D. Reasor, and A. C. Didlake Jr., 2016: The rapid intensification of Hurricane Karl (2010): New remote sensing observations of convective bursts from the Global Hawk platform. J. Atmos. Sci., 73, 3617-3639, https://doi.org/10.1175/JAS-D-16-0026.1.

Hazelton, A. T., R. F. Rogers, and R. E. Hart, 2015: Shear-relative asymmetries in tropical cyclone eyewall slope. Mon. Wea. Rev., 143, 883-903, https://doi.org/10.1175/MWR-D-14-00122.1.

- — , and — 2017a: Analyzing simulated convective bursts in two Atlantic hurricanes. Part I: Burst formation and development. Mon. Wea. Rev., 145, 3073-3094, https://doi.org/ 10.1175/MWR-D-16-0267.1.

— R. E. Hart, and R. F. Rogers, 2017b: Analyzing simulated convective bursts in two Atlantic hurricanes. Part II: Intensity change due to bursts. Mon. Wea. Rev., 145, 3095-3117, https:// doi.org/10.1175/MWR-D-16-0268.1.

Hendricks, E. A., M. S. Peng, B. Fu, and T. Li, 2010: Quantifying environmental control on tropical cyclone intensity change. Mon. Wea. Rev., 138, 3243-3271, https://doi.org/10.1175/ 2010MWR3185.1.

Hock, T. F., and J. L. Franklin, 1999: The NCAR GPS dropwindsonde. Bull. Amer. Meteor. Soc., 80, 407-420, https://doi.org/ 10.1175/1520-0477(1999)080<0407:TNGD>2.0.CO;2.

Kaplan, J., and M. DeMaria, 2003: Large-scale characteristics of rapidly intensifying tropical cyclones in the North Atlantic basin. Wea. Forecasting, 18, 1093-1108, https://doi.org/10.1175/ 1520-0434(2003)018<1093:LCORIT>2.0.CO;2.

Kepert, J. D., 2001: The dynamics of boundary layer jets within the tropical cyclone core. Part I: Linear theory. J. Atmos. Sci., 58, 2469-2484, https://doi.org/10.1175/1520-0469(2001)058<2469: TDOBLJ $>2.0 . \mathrm{CO} ; 2$.

2006a: Observed boundary layer wind structure and balance in the hurricane core. Part I: Hurricane Georges. J. Atmos. Sci., 63, 2169-2193, https://doi.org/10.1175/JAS3745.1.

2006b: Observed boundary layer wind structure and balance in the hurricane core. Part II: Hurricane Mitch. J. Atmos. Sci., 63, 2194-2211, https://doi.org/10.1175/JAS3746.1.

— 2010: Slab- and height-resolving models of the tropical cyclone boundary layer. Part I: Comparing the simulations. Quart. J. Roy. Meteor. Soc., 136, 1686-1699, https://doi.org/ 10.1002/qj.667.

2013: How does the boundary layer contribute to eyewall replacement cycles in axisymmetric tropical cyclones? J. Atmos. Sci., 70, 2808-2830, https://doi.org/10.1175/ JAS-D-13-046.1.

and Y. Wang, 2001: The dynamics of boundary layer jets within the tropical cyclone core. Part II: Nonlinear enhancement. 
J. Atmos. Sci., 58, 2485-2501, https://doi.org/10.1175/ 1520-0469(2001)058<2485:TDOBLJ $>2$.0.CO;2.

_- J. Schwendike, and H. Ramsay, 2016: Why is the tropical cyclone boundary layer not "well mixed"? J. Atmos. Sci., 73, 957-973, https://doi.org/10.1175/JAS-D-15-0216.1.

Klotz, B. W., and E. W. Uhlhorn, 2014: Improved stepped frequency microwave radiometer tropical cyclone surface winds in heavy precipitation. J. Atmos. Oceanic Technol., 31, 23922408, https://doi.org/10.1175/JTECH-D-14-00028.1.

Landsea, C. W., and J. L. Franklin, 2013: Atlantic hurricane database uncertainty and presentation of a new database format. Mon. Wea. Rev., 141, 3576-3592, https://doi.org/10.1175/ MWR-D-12-00254.1.

LeeJoice, R. N., 2000: Hurricane inner-core structure as revealed by GPS dropwindsondes. M.S. thesis, Dept. of Atmospheric Science, Colorado State University, $56 \mathrm{pp}$.

Leipper, D. F., and D. Volgenau, 1972: Hurricane heat potential of the Gulf of Mexico. J. Phys. Oceanogr., 2, 218-224, https://doi.org/ 10.1175/1520-0485(1972)002<0218:HHPOTG > 2.0.CO;2.

Möller, J. D., and M. T. Montgomery, 2000: Tropical cyclone evolution via potential vorticity anomalies in a three-dimensional balance model. J. Atmos. Sci., 57, 3366-3387, https://doi.org/ 10.1175/1520-0469(2000)057<3366:TCEVPV>2.0.CO;2.

Montgomery, M. T., and R. J. Kallenbach, 1997: A theory for vortex Rossby-waves and its application to spiral bands and intensity changes in hurricanes. Quart. J. Roy. Meteor. Soc., 123, 435-465, https://doi.org/10.1002/qj.49712353810.

, M. M. Bell, S. D. Aberson, and M. L. Black, 2006: Hurricane Isabel (2003): New insights into the physics of intense storms. Part I: Mean vortex structure and maximum intensity estimates. Bull. Amer. Meteor. Soc., 87, 1335-1347, https://doi.org/ 10.1175/BAMS-87-10-1335.

— , R. K. Smith, and S. V. Nguyen, 2010: Sensitivity of tropicalcyclone models to the surface drag coefficient. Quart. J. Roy. Meteor. Soc., 136, 1945-1953, https://doi.org/10.1002/qj.702.

Nguyen, L. T., R. F. Rogers, and P. D. Reasor, 2017: Thermodynamic and kinematic influences on precipitation symmetry in sheared tropical cyclones: Bertha and Cristobal (2014). Mon. Wea. Rev., 145, 4423-4446, https://doi.org/10.1175/ MWR-D-17-0073.1.

Nolan, D. S., and L. D. Grasso, 2003: Nonhydrostatic, threedimensional perturbations to balanced, hurricane-like vortices. Part II: Symmetric response and nonlinear simulations. J. Atmos. Sci., 60, 2717-2745, https://doi.org/10.1175/ 1520-0469(2003)060<2717:NTPTBH >2.0.CO;2.

—, and M. T. Montgomery, 2002: Nonhydrostatic, threedimensional perturbations to balanced, hurricane-like vortices. Part I: Linearized formulation, stability, and evolution. J. Atmos. Sci., 59, 2989-3020, https://doi.org/10.1175/ 1520-0469(2002)059<2989:NTDPTB > 2.0.CO;2.

—, Y. Moon, and D. P. Stern, 2007: Tropical cyclone intensification from asymmetric convection: Energetics and efficiency. J. Atmos. Sci., 64, 3377-3405, https://doi.org/10.1175/JAS3988.1.

Ooyama, K., 1969: Numerical simulation of the life cycle of tropical cyclones. J. Atmos. Sci., 26, 3-40, https://doi.org/ 10.1175/1520-0469(1969)026<0003:NSOTLC >2.0.CO;2.

Paterson, L. A., B. N. Hanstrum, N. E. Davidson, and H. C. Weber, 2005: Influence of environmental vertical wind shear on the intensity of hurricane-strength tropical cyclones in the Australian region. Mon. Wea. Rev., 133, 3644-3660, https://doi.org/10.1175/ MWR3041.1.

Pendergrass, A. G., and H. E. Willoughby, 2009: Diabatically induced secondary flows in tropical cyclones. Part I: Quasi-steady forcing. Mon. Wea. Rev., 137, 805-821, https://doi.org/10.1175/ 2008MWR2657.1.

Persing, J., and M. T. Montgomery, 2003: Hurricane superintensity. J. Atmos. Sci., 60, 2349-2371, https://doi.org/10.1175/ 1520-0469(2003)060<2349:HS > 2.0.CO;2.

Powell, M. D., P. J. Vickery, and T. A. Reinhold, 2003: Reduced drag coefficient for high wind speeds in tropical cyclones. Nature, 422, 279-283, https://doi.org/10.1038/nature01481.

Reasor, P. D., R. Rogers, and S. Lorsolo, 2013: Environmental flow impacts on tropical cyclone structure diagnosed from airborne Doppler radar composites. Mon. Wea. Rev., 141, 2949-2969, https://doi.org/10.1175/MWR-D-12-00334.1.

Riehl, H., 1954: Tropical Meteorology. McGraw-Hill, 392 pp.

Rogers, R., and E. Uhlhorn, 2008: Observations of the structure and evolution of surface and flight-level wind asymmetries in Hurricane Rita (2005). Geophys. Res. Lett., 35, L22811, https:// doi.org/10.1029/2008GL034774.

— , and Coauthors, 2006: The Intensity Forecasting Experiment: A NOAA multiyear field program for improving tropical cyclone intensity forecasts. Bull. Amer. Meteor. Soc., 87, 15231537, https://doi.org/10.1175/BAMS-87-11-1523.

—, S. Lorsolo, P. Reasor, J. Gamache, and F. Marks, 2012: Multiscale analysis of tropical cyclone kinematic structure from airborne Doppler radar composites. Mon. Wea. Rev., 140, 77-99, https://doi.org/10.1175/MWR-D-10-05075.1.

_, P. Reasor, and S. Lorsolo, 2013a: Airborne Doppler observations of the inner-core structural differences between intensifying and steady-state tropical cyclones. Mon. Wea. Rev., 141, 2970-2991, https://doi.org/10.1175/MWR-D-12-00357.1.

_ casting Experiment: A progress report. Bull. Amer. Meteor. Soc., 94, 859-882, https://doi.org/10.1175/BAMS-D-12-00089.1.

- P. D. Reasor, and J. A. Zhang, 2015: Multiscale structure and evolution of Hurricane Earl (2010) during rapid intensification. Mon. Wea. Rev., 143, 536-562, https://doi.org/ 10.1175/MWR-D-14-00175.1.

—, J. A. Zhang, J. Zawislak, H. Jiang, G. R. Alvey III, E. J. Zipser, and S. N. Stevenson, 2016: Observations of the structure and evolution of Hurricane Edouard (2014) during intensity change. Part II: Kinematic structure and the distribution of deep convection. Mon. Wea. Rev., 144, 3355-3376, https://doi.org/10.1175/ MWR-D-16-0017.1.

Rosenthal, S. L., 1971: The response of a tropical cyclone model to variations in boundary layer parameters, initial conditions, lateral boundary conditions, and domain size. Mon. Wea. Rev., 99, 767-777, https://doi.org/10.1175/1520-0493(1971)099<0767: TROATC $>2.3 . \mathrm{CO} ; 2$.

Schubert, W. H., and J. J. Hack, 1982: Inertial stability and tropical cyclone development. J. Atmos. Sci., 39, 1687-1697, https:// doi.org/10.1175/1520-0469(1982)039<1687:ISATCD>2.0.CO;2.

Schwendike, J., and J. D. Kepert, 2008: The boundary layer winds in Hurricanes Danielle (1998) and Isabel (2003). Mon. Wea. Rev., 136, 3168-3192, https://doi.org/10.1175/2007MWR2296.1.

Shapiro, L. J., 2000: Potential vorticity asymmetries and tropical cyclone evolution in a moist three-layer model. J. Atmos. Sci., 57, 3645-3662, https://doi.org/10.1175/1520-0469(2000)057<3645: PVAATC $>2.0 . \mathrm{CO} ; 2$.

_ , and H. E. Willoughby, 1982: The response of balanced hurricanes to local sources of heat and momentum. J. Atmos. Sci., 39, 378-394, https://doi.org/10.1175/1520-0469(1982)039<0378: TROBHT $>2.0 . \mathrm{CO} ; 2$.

Smith, R. K., and M. T. Montgomery, 2016: The efficiency of diabatic heating and tropical cyclone intensification. Quart. 
J. Roy. Meteor. Soc., 142, 2081-2086, https://doi.org/10.1002/ qj.2804.

- , - and N. Van Sang, 2009: Tropical cyclone spin-up revisited. Quart. J. Roy. Meteor. Soc., 135, 1321-1335, https:// doi.org/10.1002/qj.428.

-,- , and G. L. Thomsen, 2014: Sensitivity of tropical-cyclone models to the surface drag coefficient in different boundarylayer schemes. Quart. J. Roy. Meteor. Soc., 140, 792-804, https:// doi.org/10.1002/qj.2057.

Snyder, J. P., 1987: Map projections: A working manual. Professional Paper 1395, U.S. Geological Survey, https://doi.org/ 10.3133/pp1395.

Stern, D. P., J. R. Brisbois, and D. S. Nolan, 2014: An expanded dataset of hurricane eyewall sizes and slopes. J. Atmos. Sci., 71, 2747-2762, https://doi.org/10.1175/JAS-D-13-0302.1.

Uhlhorn, E. W., and P. G. Black, 2003: Verification of remotely sensed sea surface winds in hurricanes. J. Atmos. Oceanic Technol., 20, 99-116, https://doi.org/10.1175/1520-0426(2003)020<0099: VORSSS $>2.0 . \mathrm{CO} ; 2$.

_, - - J. L. Franklin, M. Goodberlet, J. Carswell, and A. S. Goldstein, 2007: Hurricane surface wind measurements from an operational stepped frequency microwave radiometer. Mon. Wea. Rev., 135, 3070-3085, https://doi.org/ 10.1175/MWR3454.1.

Vigh, J. L., and W. H. Schubert, 2009: Rapid development of the tropical cyclone warm core. J. Atmos. Sci., 66, 3335-3350, https://doi.org/10.1175/2009JAS3092.1.
Willoughby, H. E., and M. B. Chelmow, 1982: Objective determination of hurricane tracks from aircraft observations. Mon. Wea. Rev., 110, 1298-1305, https://doi.org/10.1175/ 1520-0493(1982)110<1298:ODOHTF>2.0.CO;2.

Zawislak, J., H. Jiang, G. R. Alvey III, E. J. Zipser, R. F. Rogers, J. A. Zhang, and S. N. Stevenson, 2016: Observations of the structure and evolution of Hurricane Edouard (2014) during intensity change. Part I: Relationship between the thermodynamic structure and precipitation. Mon. Wea. Rev., 144, 3333-3354, https://doi.org/10.1175/ MWR-D-16-0018.1.

Zhang, F., and K. Emanuel, 2016: On the role of surface fluxes and WISHE in tropical cyclone intensification. J. Atmos. Sci., 73, 2011-2019, https://doi.org/10.1175/JAS-D-16-0011.1.

Zhang, J. A., R. F. Rogers, D. S. Nolan, and F. D. Marks Jr., 2011: On the characteristic height scales of the hurricane boundary layer. Mon. Wea. Rev., 139, 2523-2535, https://doi.org/10.1175/ MWR-D-10-05017.1.

, P. D. Reasor, E. W. Uhlhorn, and F. D. Marks Jr., 2013: Asymmetric hurricane boundary layer structure from dropsonde composites in relation to the environmental vertical wind shear. Mon. Wea. Rev., 141, 3968-3984, https://doi.org/ 10.1175/MWR-D-12-00335.1.

_ _ _ and V. Tallapragada, 2017: Impact of parameterized boundary layer structure on tropical cyclone rapid intensification forecasts in HWRF. Mon. Wea. Rev., 145, 1413-1426, https:// doi.org/10.1175/MWR-D-16-0129.1. 